\title{
Integrating the care of the complex COPD patient
}

\section{Proceedings of the European Seminars in Respiratory Medicine course, Long-Term Integrated Care of COPD Patients held in Stresa, Italy, on 16-17 June, 2016}

\section{Claudio F. Donner1, Laura Carrozzi2,3, Sara Maio4,5, Sandra Baldacci4,5, Francesco Pistelli6, Giovanni Viegi4,5, Andrea Purro7, Roberto Torchio8, Enrico Clini9, Sandro Amaducci10, Roger Goldstein11, Michael Morgan12, Jean Bourbeau13, Guido Vagheggini14, Emiel F.M. Wouters ${ }^{15}$, Richard L. ZuWallack16}

1 Mondo Medico, Multidisciplinary and Rehabilitation Outpatient Clinic, Borgomanero (NO), Italy

2 Department of Surgical, Medical, Molecular Pathology and Critical Area, University of Pisa, Italy

3 Pulmonary Unit, University Hospital of Pisa, Italy

4 CNR-Institute of Biomedicine and Molecular Immunology “A. Monroy”, Palermo, Italy

5 CNR-Institute of Clinical Physiology, Pisa, Italy

6 Unit of Pulmonology, Cardio-Thoracic and Vascular Department, University Hospital of Pisa, Italy

7 Department of Emergency and Critical Care Medicine, Humanitas Gradenigo Hospital, Turin, Italy

8 Respiratory Function and Sleep Laboratory, University Hospital S. Luigi Gonzaga, Orbassano (TO) Italy

9 Department of Medical and Surgical Sciences, University of Modena-Reggio Emilia, Modena, Italy

10 Pneumology Department, San Carlo Borromeo Hospital, Milan, Italy

11 West Park Healthcare Centre and University of Toronto, Canada

12 University Hospitals of Leicester NHS Trust, UK

13 Center for Innovative Medicine, McGill University Health Centre Montreal, QC, Canada

14 Volterra Ricerche Foundation, Weaning and Pulmonary Rehabilitation Unit, Auxilium Vitae

Rehabilitation Centre, Volterra, Italy

15 Pulmonology, School of Nutrition and Translational Research in Metabolism, Faculty of Health, Medicine and Life Sciences, University of Maastricht, The Netherlands

16 St. Francis Hospital Hartford, CT and University of CT School of Medicine Farmington, CT, USA

Corresponding author: Claudio F. Donner, Mondo Medico di I.F.I.M. srl, Multidisciplinary and Rehabilitation Outpatient Clinic, Via Monsignor Cavigioli 10, 28021 Borgomanero (NO), Italy.

Tel. +39.0322.836718 - Fax +39.0322.869950.

E-mail: cfdonner@mondomedico.it

Key words: European Seminars in Respiratory Medicine; Long-Term Integrated Care; COPD.

Received for publication: 27 October 2016

Accepted for publication: 7 November 2016

(C) Copyright C.F. Donner et al., 2017

Tipografia PI-ME Editrice, Italy

Monaldi Archives for Chest Disease Pulmonary Series 2017; $87: 786$

doi: 10.4081/monaldi.2017.786

This article is distributed under the terms of the Creative Commons Attribution Noncommercial License (by-nc 4.0) which permits any noncommercial use, distribution, and reproduction in any medium, provided the original author(s) and source are credited.

\section{Introduction}

The European Seminars in Respiratory Medicine has represented an outstanding series updating new science in respiratory disease from the 1990 's up to the early beginning of this $21^{\text {st }}$ century $[1,2]$. Its aim is to update issues and current science, focusing on the multidisciplinary approach to patients with respiratory disease. As such, it represents a unique opportunity for specialists in Respiratory Medicine involved in Basic and Clinical Research to discuss topical and debated problems in medical care, at a top level forum guided by an expert panel of authors.

The structure of the seminar is based on the following pillars:

- Attendance at the Seminars is strictly limited: selection of participants is based, in order of priority, on scientific curriculum, age (younger specialists are privileged), and early receipt of the application form.

- Each topic is allotted considerable time for presentation and discussion. The first section is devoted to a series of presentations (with adequate time allocated for discussion) by an expert panel of researchers and clinicians. In the second section involves discussions of controversial issues, in a smaller audience format encouraging interaction between the panel and audience.

- "Meet the expert" seminars discuss topical subjects in more depth, utilizing an interactive tutorial. 
The Seminar is accredited to provide continuing medical education (CME) for Italian physicians, with approval of up to 100 participants, providing $10 \mathrm{CME}$ credits to professionals in the following specialties: Allergology and Immunology, Anesthesiology. Cardiology, Geriatrics, General Practice, Internal Medicine, Pulmonology, Radiology, Rehabilitation, Rheumatology, and Thoracic Surgery.

The recent epidemiologic trend, placing chronic obstructive pulmonary disease (COPD) as the third-leading cause of death worldwide, underscores the huge burden placed on society by this disease. This includes disability, higher mortality risk, direct health service costs (including pharmaceutical, inpatient, outpatient, and supplemental oxygen costs), as well as prominent indirect costs (including lost production and burden on families) [3]. The prevalence COPD, its social and economic burdens, and the complexity of the problems present in the patient with advanced disease necessitate a new and more structured approach to the long term management of this disease.

The objectives of this Seminar on "The Long Term Integrated Care of COPD Patients" held in Stresa, Italy, from 16-17 June, 2016, were:

- Description of the concepts of integrated care and chronic disease management.

- Discussion of specific components of disease management as they may apply to the COPD patient.

- Provision of several innovative examples of COPD disease management programs originating from different health care systems. The scientific contents of this seminar focused on the optimal care of the COPD patient. Optimizing care for the complex COPD patient requires: i) An individualized, patient-centered approach, recognizing and treating all aspects of the respiratory disease, its systemic effects and common co-morbidities; and ii) integrating medical care among health care professionals and across the health care sector. Optimal integration of medical care for COPD is still in its infancy, and its implementation will undoubtedly represent a paradigm shift in our thinking. In particular, the scientific program of this Seminar described in detail the specific components of disease management that apply to the COPD patient, providing innovative examples programs originating from different health care systems, providing an opportunity to compare approaches implemented in different countries.

\section{Prevalence of COPD}

\section{Key points}

- A high and growing prevalence of COPD, both globally and regionally, with substantial variation in trends between different regions is observed

- The prevalence of respiratory symptoms/diseases is still increasing in the Italian general population

- Occupational exposure and living in an urban area are related to asthmatic and/or allergic symptoms/diagnosis

- Smoking habits, occupational exposure and living in an urban area are related to bronchitic symptoms

\section{Prevalence and incidence}

The prevalence of a disease measures the number of cases of the disease in a population at a given time, even though the process of the data collection may take place over days, weeks, or years. It is calculated as the number of people with the disease divided by the total population at the time of data collection, usually expressed as a percentage [3].The prevalence rate has to be collected through cross-sectional studies: it provides a static measure; it estimates the burden of the disease; and it can be useful for public health research and decision-making [3-5].
Unlike prevalence, the incidence of a disease measures the risk of developing new disease within a specified period of time. Annual incidence is calculated as the number of new cases occurring in 12 months divided by the population who was diseases-free at the beginning of the period. Incidence can be hard to measure as it involves knowing subjects who are disease-free at the beginning of the period; a prospective study design is needed [3,4].

Prevalence and incidence are interrelated. In particular, the prevalence depends on disease incidence and duration. A high prevalence may be due to a high incidence, and/or a long duration of the disease; an increase in prevalence over time may be due to an increase in incidence, or a longer duration of the disease (i.e., longer survival) $[4,5]$.

\section{Definition of COPD matters in prevalence studies}

Different criteria can be used to define COPD, and this methodological aspect is relevant to epidemiology, when data from different studies and populations have to be compared or pooled together [6,7]. For spirometry-defined COPD, the disease prevalence in a general population depends on the threshold used for determining airflow limitation [6]. A definition based on a fixed value of $\mathrm{FEV}_{1} / \mathrm{FVC}(<0.70)$ overestimates COPD in the elderly, and under-underestimates it in the younger people. To define COPD as a $\mathrm{FEV}_{1} / \mathrm{FVC}$ ratio below the lower limit of normal (LLN) takes into account the age-related changes in lung function $[8,9]$.

\section{Prevalence of COPD \\ COPD global prevalence}

A recent review estimates COPD prevalence for the years 1990 and 2010 across six world regions, based on the best available evidence in publicly accessible scientific databases [10]. In 123 selected studies of 52 countries, the global prevalence of spirometry-defined COPD $(93 \%$ defined by $\mathrm{FEV}_{1} / \mathrm{FVC}<0.70$ ) in subjects over 30 years of age was $11.3 \%$ in the year 2010. The highest prevalence was found in the Americas region, followed by Eastern Mediterranean and European regions, whereas the lowest in the South-East Asian region. The prevalence was higher in men (14.3\%) than in women (7.6\%), in high than in lowmiddle income countries (12.0 vs 10.6), in urban than in rural areas (13.2 vs 10.2) [10]. About 227 million COPD cases were estimated in 1990 (global prevalence 10.7\%); the number of estimated case increased to 384 million in 2010 . This $68.9 \%$ increase is mainly driven by global demographic changes over the 20 years period; however, other factors underline the current trend. The lowest increase in total number of COPD cases in the European region may reflect reduction in prevalence of smoking in Europe; gender related differences among the different regions are evident [10].

Prospective studies conducted in elderly population in Europe [11,12] showed that the age-specific incidence of COPD increased with age until 80 years. The incidence increased by age also in never smoking older subjects [11]: the detection of new cases in never smokers indicates that additional factors may independently contribute to COPD development, mainly environmental conditions (outdoor and indoor pollution, climate changes).

\section{Prevalence of COPD from Italian population-based studies}

The Pulmonary Environmental Epidemiology Unit of the Institute of Clinical Physiology (EPAP-IFC) of the National Research Council (CNR) started to carry out epidemiological surveys on general population samples in Italy in the ' 80 s. In particular, the main objective was to 
study the natural history of COPD in two general population samples living in Northern (Po river Delta area - PD) and Central (Pisa urban and suburban area - PI) Italy, in relation to risk factors such as smoking and environmental pollution.

Changes in prevalence of respiratory symptoms/diseases between the first and the second cross-sectional surveys, in subjects living in rural and urban area, were analyzed: the highest variations were shown in PD subjects under 25 years, especially for wheeze (1st-2nd survey prevalence: $6.0-17.0 \%$ in males, $4.0-12.0 \%$ in females), attacks of wheeze (3.0-6.0\% in males), dyspnea (2.0-6.0\% in males, 3.0 $11.0 \%$ in females) and cough (5.0-10.0\% in females), which increased two to three fold. In PI, only wheeze in both sexes increased (14.0-22.0\% in males, $10.0-15.0 \%$ in females) [13]. A different pattern between sexes was observed in subjects aged 25-64 years: wheeze and dyspnea consistently increased in PD men (14.0-27.0\% and $13.0-17.0 \%$, respectively), whilst cough $(7.0-11.0 \%$ in PD and $11.0-15.0 \%$ in PI), phlegm (6.0-11.0\% in PD and 7.0-10.0\% in PI) and wheeze (7.0-14.0\% in PD and $13.0-19.0 \%$ in PI) increased in women in both areas. Moreover, PI females showed a significant increase in dyspnea (19.0-29.0\%) [13]. Recent analyses on PI sample showed that the adjusted prevalence rates of asthma attacks, allergic rhinitis (AR) and COPD increased in a period of 25 years: current asthma at-

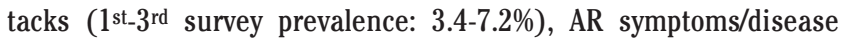
(16.2-37.4\%), usual phlegm (8.7-19.5\%) and COPD (2.1-6.8\%) more than doubled from PI1 to PI3. Fewer increases were shown for usual cough and airway obstruction (A0) (11.4-16.3\%, 10.8-21.1\%, respectively) [14].

A generalized estimating equations (GEE) model showed significant relationships between asthmatic and/or allergic symptoms/diagnosis and occupational exposure: a $20 \%$ higher risk of asthma and attacks of asthma and $40 \%$ higher risk of AR in subjects exposed to fumes/gases/dusts at work, with respect to unexposed subjects. Living in an urban area, with respect to living in a suburban one, was associated to a $20 \%$ higher risk of AR [14]. Smoking habits, occupational exposures and living in an urban area were related to bronchitic symptoms/diagnosis. In particular, subjects exposed at work had a higher risk of usual cough, usual phlegm and COPD with respect to unexposed subjects (25\%, 40\% and $80 \%$ higher risk, respectively). Heavy smoking habit ( $\geq 24$ pack-years), with respect to non smoking, was associated with a nearly $340 \%$ higher risk of bronchitic symptoms/diagnosis; a nearly $170 \%$ higher risk was shown for A0. At last, living in an urban area, with respect to living in a suburban one, was related to a higher risk of usual phlegm (30\%) and COPD (50\%) [14].

These data confirm the increasing trend in asthmatic and allergic diseases/symptoms found in other national and international studies [15-17]. With regard to the COPD symptoms/diagnosis trend, repeated cross-sectional studies reported inconsistent results so far; our study added new evidences about the COPD increase in the last decades, confirming data from a Norwegian study that show an increasing prevalence of spirometry-defined COPD (i.e. $\mathrm{FEV}_{1} / \mathrm{FVC}$ $<0.70$ ) from $7 \%$ to $14 \%$ in nine years (15). Instead, Spanish and Swedish studies reported a decreasing trend in the prevalence of COPD over $10-15$ years periods (from $9.1 \%$ to $4.5 \%$ and from $9.5 \%$ to $6.3 \%$, respectively) [18].

Urban living is a risk factor for allergic rhinitis and COPD symptoms/diagnosis, confirming our previous findings obtained through a geographic information system approach [19]; moreover, the current data are in line with our previous study showing that urban living is associated with higher bronchial hyper-responsiveness [20] and in agreement with an American Thoracic Society statement highlighting the relationship between outdoor pollution and COPD development [21].

\section{An overview of COPD}

\section{Key points}

- $5-10 \%$ of adults aged over 40 years have COPD, with a higher prevalence in men than women

- The key risk factor for chronic obstructive pulmonary disease is tobacco smoke, but occupational exposures, pollution and genetic factors play a role

- The most important symptoms of chronic obstructive pulmonary disease are breathlessness on exertion and chronic cough with or without phlegm, but fatigue, anorexia and weight loss can arise as the disease progresses

- Treatment of COPD is multimodal, including smoking cessation, medical treatment with bronchodilators as well as inhibitors of inflammation, physical exercise and oxygen therapy

COPD is characterized by persistent airflow limitation that is usually progressive and associated with a chronic inflammatory response in the airways and lungs to noxious particles or gases [22]. The persistent airflow limitation results from a combination of diffuse small airway disease and destruction of the lung parenchyma (emphysema) [23]. Chronic bronchitis is defined clinically as the presence of a chronic productive cough for 3 months during each of 2 consecutive years (other causes of cough excluded). Emphysema is defined pathologically as an abnormal, permanent enlargement of the air spaces distal to the terminal bronchioles, accompanied by destruction of their walls and without obvious fibrosis [24].

COPD affects 32 million persons in the United States and is the third leading cause of death in that country. The National Health Interview Survey reports the prevalence of emphysema at 18 cases per 1000 persons and chronic bronchitis at 34 cases per 1000 persons [25]. Overall, $5-10 \%$ of adults aged over 40 years in Europe have COPD, with a higher prevalence in men than women. The mortality rate, age-standardized to the European Standard Population, is about 18 per 100000 inhabitants per year [26]. COPD does occur in individuals who have never smoked [27]. Although the role of air pollution in the etiology of COPD is unclear, its effect is small compared with that of cigarette smoking [28]. In developing countries, the use of biomass fuels with indoor cooking and heating is a major contributor to the worldwide prevalence of COPD. Long-term exposure to traffic-related air pollution may also be a factor in COPD in patients with diabetes and asthma [29,30]. The Dutch hypothesis postulates that individuals with nonspecific airway hyperreactivity who smoke cigarettes have an accelerated decline in lung function and, therefore, are at a particularly high risk of developing COPD. Nonspecific airway hyperreactivity is inversely related to $\mathrm{FEV}_{1}$ predicts a more rapid decline in lung function. However, the potential role of airway hyperresponsiveness as a risk factor in the development of COPD in smokers is still unclear. Moreover, bronchial hyperreactivity may result from airway inflammation observed with the development of smoking-related chronic bronchitis. This may contribute to airway remodeling, leading to a fixed obstruction as is seen in COPD. Alpha-1-antitrypsin (AAT) is a glycoprotein member of the serine protease inhibitor family that is synthesized in the liver and is secreted into the bloodstream. This 394-amino-acid, single-chain protein neutralizes neutrophil elastase in the lung interstitium, thereby protecting it from elastolytic breakdown. Severe AAT deficiency predisposes to unopposed elastolysis, with the clinical sequela of early-onset of panacinar emphysema. AAT deficiency is the only known major genetic risk factor for developing COPD, accounting for somewhat less 
than $1 \%$ of all cases in the United States. Severe deficiency leads to premature emphysema at an average age of 53 years for nonsmokers and 40 years for smokers. Nearly 24 variants of the AAT molecule have been identified; all are inherited as co-dominant alleles.

COPD is a syndrome with several phenotypes. Patients typically present with a combination of signs and symptoms of chronic bronchitis, emphysema, and reactive airway disease. Symptoms include the following: i) Cough, usually worse in the morning and productive of a small amount of colorless sputum; ii) Acute chest illness (exacerbations); iii) Breathlessness: this is usually the most significant symptom, but usually does not occur until the sixth decade of life; iv) Wheezing: this may occur in some patients, particularly during exertion and exacerbations.

The severity of airflow obstruction remains a primary means of staging COPD since the American Thoracic Society (ATS) provided criteria for staging COPD based on: i) defining the presence of obstruction (ratio of $\mathrm{FEV}_{1}$ to forced vital capacity $\left[\mathrm{FEV}_{1} / \mathrm{FVC}\right]<70 \%$ ); and ii) determining its severity based on percent-predicted $\mathrm{FEV}_{1}$. ATS and Global Initiative for Chronic Obstructive Lung Disease (GOLD) criteria for assessing the severity of airflow obstruction (based on the percent predicted post-bronchodilator $\mathrm{FEV}_{1}$ when the $\mathrm{FEV}_{1} / \mathrm{FVC}$ is $<70 \%$ ) are as follows:

- Stage I (mild) - $\mathrm{FEV}_{1} 80 \%$ or greater of predicted.

- Stage II (moderate) - $\mathrm{FEV}_{1} 50-79 \%$ of predicted.

- Stage III (severe) - $\mathrm{FEV}_{1} 30-49 \%$ of predicted.

- Stage IV (very severe) - $\mathrm{FEV}_{1}$ less than $30 \%$ of predicted or $\mathrm{FEV}_{1}$.

However, staging systems based on spirometry have limited utility in predicting mortality. The recognition that COPD is a systemic disease has helped in developing criteria that are better at predicting mortality than is assessment of airway obstruction alone. A widely-used system for COPD prognosis is the BODE index (body mass index, obstruction [ $\mathrm{FEV}_{1}$ ], dyspnea [modified Medical Research Council dyspnea scale], and exercise capacity [six minute walk distance]) [31].

Smoking cessation continues to be the most important therapeutic intervention for COPD. Risk factor reduction (e.g., influenza vaccine) is appropriate for all stages of COPD. Other therapeutic approaches to management by stage include the following [23]:

- Stage I (mild obstruction): Short-acting bronchodilator as-needed.

- Stage II (moderate obstruction): Short-acting bronchodilator asneeded; long-acting bronchodilator(s); cardiopulmonary rehabilitation.

- Stage III (severe obstruction): Short-acting bronchodilator asneeded; long-acting bronchodilator(s); cardiopulmonary rehabilitation; inhaled glucocorticoids if repeated exacerbations.

- Stage IV (very severe obstruction or moderate obstruction with evidence of chronic respiratory failure): Short-acting bronchodilator as needed; long-acting bronchodilator(s); cardiopulmonary rehabilitation; inhaled glucocorticoids if repeated exacerbations; long-term oxygen therapy (if criteria are met); consider surgical options such as lung volume reduction surgery (LVRS) and lung transplantation. COPD is commonly associated with progressive hypoxemia. Oxygen administration reduces mortality rates in patients with advanced COPD [32], probably because of its favourable effects on pulmonary hemodynamics. Noninvasive positive pressure ventilation can provide significant benefits in selected patients with acute hypercapnic respiratory failure due to $\mathrm{COPD}$, including a reduction in the need for endotracheal intubation, reduced hospital stay, and a mortality benefit [33].

Influenza and pneumococcal vaccination is a safe and effective modality to improve outcome in COPD $[34,35]$, presumably due to reducing infections in susceptible patients. The pneumococcal vaccine should be offered to all patients older than 65 years or to patients of any age who have an $\mathrm{FEV}_{1}$ of less than $40 \%$ of predicted. The influenza vaccine should be given annually to all COPD patients.

Many patients with COPD are unable to enjoy life to the fullest because of shortness of breath, physical limitations, and inactivity. Pulmonary rehabilitation encompasses an array of therapeutic modalities designed to improve the patient's quality of life by decreasing airflow limitation, preventing secondary medical complications, and alleviating respiratory symptoms. Successful implementation of a pulmonary rehabilitation program usually requires a team approach, with individual components provided by healthcare professionals who have experience in managing COPD (e.g., physician, dietitian, nurse, respiratory therapist, exercise physiologist, physical therapist, occupational therapist, recreational therapist, cardiorespiratory technician, pharmacist, psychosocial professionals). In COPD, pulmonary rehabilitation has documented benefits in exercise tolerance, dyspnea, and health related quality of life. Additionally, it may reduce health care utilization [36].

Given the generally progressive nature of COPD and the fact that it is the third leading cause of death world-wide, clinicians should always include end-of-life care discussions in their visits with patients with advanced stages of this disease. These discussions should focus on palliative efforts to improve quality of life, as well as assistance with advanced directives, advanced care planning, and referrals for hospice and home care when needed.

\section{COPD and chronic heart disease}

\section{Key points}

- Patients with COPD and heart disease share a common risk factor: cigarette smoking

- The use of a fixed $\mathrm{FEV}_{1} / \mathrm{FVC}$ ratio of $<0.70$ to detect airway obstruction instead of using lower limit of normal thresholds, can lead to a misdiagnosis of COPD, especially in the elderly, those with heart failure and those with low lung volumes; the consequent can be unjustified use of bronchodilators in these patients

- A greater extent of emphysema on CT scan and greater airflow obstruction are linearly related to impaired left ventricular filling, reduced stroke volume, and lower cardiac output

- Reducing hyperinflation in COPD with long-acting bronchodilators results in improved cardiopulmonary performance

The heart and lungs share the same space in the thorax, and often impairment of one can produce serious effects on the other. Additionally, similar risk factors (e.g., cigarette smoking) frequently cause cardiovascular disease and COPD in the same patient. Smokers have ten times higher risk of developing lung cancer than nonsmokers and four times the risk of developing COPD [37]. In a similar way, smoking increases the risk of ischemic heart attack 1.5 times for ten cigarettes/day smokers and 2 times for 30 cigarettes/day smokers [38].

Clinical symptoms and signs of COPD and chronic heart failure (CHF) frequently overlap, so evaluation of cardiac versus pulmonary disease in daily practice is often problematic and occasionally misleading. Because of this, echocardiography as well as pulmonary function tests should be considered in every symptomatic COPD patient to avoid potential misdiagnosis and subsequent inappropriate treatment. Simply getting spirometry in patients with COPD is problematic, since - at least in one health care setting - the number of COPD patients without pulmonary function studies was greater than the number of 
CHF patients without echocardiographic examination, indicating that the typical cardiology workup is more objective than the pulmonary workup of symptomatic patients [39].

According to ERS/ATS guidelines, a COPD diagnosis requires airway obstruction confirmation by spirometry [40]. From a physiologic point of view, obstruction must be considered when a forced expiratory volume in one second to vital capacity $\left(\mathrm{FEV}_{1} / \mathrm{NC}\right)$ is below the lower limit of normal (LLN, such as $<89 \%$ in males $<88 \%$ in females) [41]. The use of a fixed ratio such as an $\mathrm{FEV}_{1} / \mathrm{NC}<70 \%$ absolute value, as utilized by GOLD guidelines, overestimates COPD occurrence in the elderly because of the age-related reduction in vital capacity, with gender differences (overestimation in males) [42]. Often in older males with heart failure and reduced lung volumes COPD group "B" could be diagnosed using fixed ratio with misdiagnosis and unjustified use of bronchodilators [43].

The prevalence of CHF in COPD patients, excluding known coronary artery disease, ranges from $0-16 \%$ in stable patients and from to $0-32 \%$ in exacerbated patients [44]. Cardiovascular diseases are correlated with COPD severity: the greater the airflow limitation the higher percentage of CHF patients with overlap of the two diseases [45]. From a different perspective, CHF patients have seven-fold relative risk of COPD.

Very severe COPD often results in cor pulmonale, with elevated pulmonary vascular resistance and secondary reductions in left ventricular (LV) filling, stroke volume, and cardiac output. A greater extent of emphysema [46] on CT scan and severer airflow obstruction are linearly related to impaired left ventricular filling, reduced stroke volume, and lower cardiac output without changes in the ejection fraction. Furthermore, hyperinflation in COPD or emphysema is associated with reduced pulmonary vein cross-sectional area, suggesting impaired LV filling. An impaired left ventricular diastolic filling pattern is, moreover, independently associated with a reduced 6 minute walking distance. So left ventricular function is impaired in patients with severe emphysema due to small end-diastolic dimensions and COPD and emphysema on CT scan are associated [47] with reduced pulmonary vein cross-sectional area. These findings suggest that impaired LV filling in COPD, and also in emphysema may be predominantly due to reduced $\mathrm{LV}$ preload from upstream pulmonary causes rather than intrinsic diastolic dysfunction, although cardio-pulmonary interactions are likely complex.

Very recently, Stone et al. [48] demonstrated that changes in cardiac structure and function could be achieved reducing lung hyperinflation. In stable, hyperinflated COPD, lung deflation with long acting bronchodilator results in structural alterations to both sides of the heart, improved biventricular stroke volume, left atrial function, and pulsatility within the pulmonary circulation. Right ventricular diastolic function and filling pressure have strong influence on the functional capacity in patients with COPD. The mechanism of the exercise intolerance is complex in COPD, thus it is difficult to identify the true contributors. Nevertheless, and right ventricular diastolic function and filling pressure have strong influence on the functional capacity in these patients [49].

Ventilation is increased in CHF patients at rest and during exercise, leading to an increase in inspiratory drive, while gas exchange is impaired in both CHF and COPD [50]. COPD had severe ventilation-perfusion mismatch, with differences between emphysema and chronic bronchitis. Both the increased heart volume in CHF and often-present pulmonary congestion and edema reduce lung volumes such as the functional residual capacity (FRC) [51]. Due to this decrease in FRC, many patients exhibit airway closure during tidal breathing, leading to ventilation-perfusion mismatch and impaired gas exchange within the lung [51].
During exercise testing it is possible to estimate ventilatory efficiency using the minute ventilation (VE) to carbon dioxide production (VC02) relationship during exercise. In heart failure there is a disproportionate increase in VE during exercise, while in COPD the relationship is variable, with disproportionate increases in VE in mild-to-moderate disease and disproportionate decreases in severe disease. More recently, it was pointed out that the intercept of the VE/VCO2 slope is related to dead space ventilation [52]. An increase in ventilatory inefficiency, as manifest by a high intercept suggests coexistent COPD in heart failure patients [53]. Nevertheless, high intercepts in patients with largely preserved spirometry (GOLD spirometric stage 1), suggest coexistent ventilation-perfusion abnormalities, explaining persistent dyspnea and activity restriction [54].

In the overlap between COPD and heart failure, skeletal muscle perfusion is reduced to a greater extent than that expected by heart failure alone. Given that these abnormalities are related closely to poor exercise tolerance, increasing skeletal muscle $\mathrm{O}_{2}$ delivery and/or decreasing $0_{2}$ demand might be of particular therapeutic relevance [55].

Overlap patients are particularly prone to respond to interventions that increase skeletal muscle $\mathrm{O}_{2}$ delivery and/or reduce $\mathrm{O}_{2}$ demand. Potential therapeutic options can, for example, include: A) Improving cardiac output by non-invasive positive pressure ventilation and/or Heliox; B) Adopting physical training strategies suitable for patients with severe central cardiorespiratory constraints (e.g., small muscle mass training); and C. Using drugs as sildenafil or nitrates to directly enhance $\mathrm{O}_{2}$ delivery-to-utilization matching with increased muscle blood flow and/or lowered $\mathrm{O}_{2}$ cost of muscle contraction [55].

Cerebral blood flow and oxygenation are generally well-preserved in COPD and increase with exercise [56]. However, in a small study, overlap patients with COPD and heart failure decreased cerebral blood flow during exercise. This drop was present despite preserved arterial oxygenation. The authors speculated that this drop in overlap patients may negatively contribute to clinically important outcomes such as exertional dyspnea, exercise tolerance, cerebrovascular disease, and cognitive impairment.

The overlap between COPD and CHF significantly reduces survival and quality of life [57], so correct and well-timed diagnosis and treatment are fundamental for the management strategies of these patients.

\section{Physical activity and long-term management of COPD patient}

\section{Key points}

- Increases in exercise capacity from physical training do not necessarily result in increases in physical activity

- Achieving a higher level of physical activity in COPD patients is arguably a preferable goal over muscle training alone

- Physical activity, a measurable variable, should be promoted in COPD patients as a beneficial lifestyle

\section{Introduction}

Physical activity (PA) is one of the major lifestyle-related health determinants [58]. Epidemiological research has already produced convincing conclusions about the health gains of being sufficiently active. However, even if the impact of PA on public health is increasingly understood in scientific and academic circles, there remains a lack of political awareness (far less than that paid to tobacco and nutrition) and recognition that comprehensive actions still need to be taken. PA is 
generally defined as any bodily movement produced by skeletal muscles that results in energy expenditure above resting level. Notwithstanding, PA is a complex behaviour that can be characterised by type, intensity, duration, patterns of activities, and symptom experience. The term 'physical activity' means many different things to different people. For public health professionals, it is a health-enhancing behaviour; others may see it as a phrase summing up a wide range of sports, leisure pursuits or active travel.

The human body evolved to move, and our physiological systems are continuously working to balance the energy we expend through PA with the energy we take in as food. A century ago, obesity was rare, as people spent far more energy in manual work and walked more for transport, and energy-dense food was less easily available. In the new century, our lifestyles have changed and chronic diseases have increased with aging population: most important, PA has been removed from our lives. Throughout childhood, PA offers opportunities to develop basic motor skills that are essential for healthy active living. As we enter old age, PA becomes a critical component of a healthy, satisfying and independent life.

\section{COPD and physical activity}

In patients with COPD PA is reduced [59], and this reduction is related to important disease characteristics, such as airway obstruction, dynamic hyperinflation, exercise intolerance, muscle weakness, systemic inflammation, and coexisting cardiovascular diseases or other comorbidities [60,61]. Furthermore, reduced levels of PA appear to be linked with impaired increased future risk of hospitalizations, and mortality [62-64]. Therefore, PA might have a central role within the disease process, so its enhancement is desirable in medical practice, and it deserves attention as a clinical outcome parameter [64]. Objective measurements of physical activity using motion detectors and validated questionnaires have also helped increased interest in this aspect of health in COPD [65].

Interestingly, it has been recently shown that PA occurs early in the natural course of the disease, with inactivity preceding both symptoms and diagnosis by around 5 years [66].

The effect of changes in PA in COPD patients as well as in elderly controls has been observed in a population-based study up to 17 years $[67,68]$. Both COPD and controls showed an increased mortality risk with low level of PA; however, improvement of PA level in those inactive COPD patients at baseline did not change this risk. This might suggest that assess and encourage PA in the early stage of the disease to maintain PA as high as possible is essential to improve prognosis.

Population-based epidemiological studies have assessed the longitudinal effect of regular PA on lung function decline or COPD incidence [69]. An inverse association between PA levels and the magnitude of lung function decline is present, although this association is not consistent across population subgroups or physical activity variables. Potential explanations for such inconsistencies include selection bias, lack of adjustment for potential confounders, and lack of consideration of changes in PA level during follow-up. International documents on COPD management suggest that PA is recommended for all patients with COPD. However, there is little COPD-specific evidence to support recommendations for PA, other than studies of pulmonary rehabilitation [70].

\section{Enhancing PA in COPD patients}

Several interventions have been considered to improve PA in the COPD population [71]. Notwithstanding, the mean achievable improvement in PA ranges from 10 to $40 \%$ of the baseline value at best, which means that strategies should be more comprehensive and adapted to the single patient. Pulmonary rehabilitation is a comprehensive intervention based on a thorough patient assessment followed by patient-tailored therapies which include, but are not limited to, exercise training, education and behavior change, and to promote the long-term adherence to health-enhancing behaviors [36]. Increases in exercise capacity in combination with behavioral change may also have the potential to increase PA in these patients [36,72,73]. Indeed, given the widened scope of rehabilitation in these patients (i.e., improve symptoms, exercise capacity, and quality of life), outcomes assessment has also broadened, allowing for the evaluation of COPD-related knowledge and self-efficacy, including PA. Transferring exercise (i.e., effective training) behavior from a supervised clinical setting to a self-directed leisure-time context poses a major challenge for most patients with COPD who completed pulmonary rehabilitation.

The practical message is that simply increasing the exercise capacity of patients with COPD is insufficient to increase participation in self-directed leisure time activity. Exercise capacity is thus considered permissive of physical activity. Behavioral, social and environmental interventions are now recognized as necessary to effect increases in PA in COPD; these include mobilizing social support, using well-established behavior change and self-regulatory techniques (self-monitoring, stimulus control, problem solving, relapse prevention management, goal setting, self-reinforcement, providing feedback on performance and developing action plans), providing higher contact time or contact frequency, and assessing and enhancing motivation to change. Last, but not least, a deeper knowledge on how COPD patients perceive symptoms limiting their PA in the every day life might help foster strategies to enhance the effectiveness of these interventions.

\section{The integrated care of the COPD patient}

\section{Key points}

- Considering the high impact of COPD on quality of life, health care utilization and mortality risk, our current medical management of this disease has considerable room for improvement

- Current medical management of COPD often focuses on just the respiratory disease, ignoring its systemic effects and common co-morbid conditions

- Current medical management of COPD is usually fragmented and uncoordinated; this is most problematic in the patient with severe disease in the peri-exacerbation period

- An interdisciplinary, patient-centered approach to medical management, along with coordination of care is necessary to improve outcomes in at-risk patients with this disease; integrated care deals with both these problem areas

\section{The burden of COPD}

Chronic obstructive pulmonary disease (COPD), besides being the third-leading cause of death worldwide, imposes a substantial symptom and functional status limitation burden on the patient and staggering expenses on health care systems. Compounding this is the fact that we do not have significant disease-modifying pharmacologic treatments, using the traditional concept of disease modification as a reduction in the rate of decline of airflow limitation [74]. However, pharmacologic and nonpharmacologic interventions can have a appreciable effect on reducing the frequency of exacerbations, which might be considered a form of disease modification [74]. While optimal management of the COPD patient must cover the entire course of the disease - and even before the disease onset in modifying risk factors such as cigarette 
smoking in young people - much of the medical management of this disease must focus, by current necessity, on the at-risk COPD patient. This discussion will deal with the high-risk patient, defined somewhat arbitrarily as that patient with a history of frequent exacerbations or in the peri-hospitalization period for an exacerbation.

\section{The at-risk COPD patient}

Despite mind-staggering advances in science and technology in the modern era, medical care for patients with chronic diseases such as COPD - at least when using re-hospitalization as a criterion - is often abysmal. For example, a landmark study by Jencks et al. [75], using 2003-2004 Medicare claims data demonstrated that almost one-fifth of discharged Medicare beneficiaries (not just COPD patients) were rehospitalized within 30 days and $34 \%$ were re-hospitalized within 90 days.

The discord between technology, on the one hand, and outcome, on the other hand, is accentuated by the fact that considerable monetary funds are spent on the medical care of elderly patients, who are most likely afflicted with chronic disease: medicare claims data in 2014 in the United States showed a peak expenditure of $\$ 43,453$ per individual in 73 year old individuals! [76].

These statistics on health care utilization have not changed substantially over the past decade or so since the publication of the Jencks article, making this problem still relevant to the hospitalized COPD patient in 2016. For example, quarterly assessments of any-cause 30-day re-admission rate for COPD in US hospitals typically range from the mid-teens to the mid-twenties. Health status is severely decreased during the COPD exacerbation, and it typically remains impaired for several weeks following the onset of the event [77]. Additionally, functional status, using directly-measured physical activity as its criterion, remains decreased for at least 2-3 weeks after onset [78]. Finally, mortality risk is increased after the first severe (i.e., hospitalized) COPD exacerbation, and goes up dramatically after the second [79].

\section{Common system problems with the COPD patient hospitalized for an exacerbation}

As stated above, the COPD patient hospitalized for an exacerbation generally has severe disease, increased symptoms, marked functional limitation, increased likelihood of having co-morbid conditions, a much greater risk of being hospitalized again in the near-future, and a higher mortality risk. Patients in the hospital and recently discharged following exacerbations have accentuated medical needs, as outlined below.

- The hospitalized COPD patient's medical care, because of the urgency of the situation, necessarily must focus on the acute problem, leaving little time for chronic disease management.

- Little patient-centered and holistic care is given in the hospital and peri-hospital period.

- Single disease guidelines often fall short in achieving desired goals in the complex, multi-morbid, hospitalized COPD patient.

- Fragmented medical care from well-meaning and accomplished practitioners is often provided.

- There is often no one health care professional to take charge throughout the hospitalization and peri-hospitalization period.

- Educating patients often takes a second place to the urgency of the situation and the need to discharge patients promptly.

- Inadequate instructions are given to the patient at almost every point in care; often these instructions are computer-generated and impersonal.

- Often the mechanism in place is inadequate for ensuring proper medications after discharge.

- No planned transition of care from the hospital, to the home, and (eventually) into the community.

\section{Reasons behind this high impact of COPD on health status, health care utilization and mortality}

There are undoubtedly multiple reasons behind the high symptom burden, functional and health status limitations, high health care utilization and high mortality in the at-risk COPD patient, as defined above. A listing of some of the problems includes:

i) The COPD patient is usually hospitalized for about 4-5 days, yet the exacerbation that led to that particular hospitalization typically lasts 3-4 weeks. Thus, the patient has not reached baseline status at the time of hospital discharge, yet the intensity of care drops off precipitously at this point in time.

ii) COPD exacerbations tend to cluster temporally [80], putting the discharged patient at-risk not only for deterioration in health status from the index exacerbation but also at high risk for a second one. In general, the strongest variable predicting a 30-day hospital readmission in COPD is a history of a hospital admission for this condition within the past year [81].

iii) The COPD patient in the peri-hospitalization period needs care based on both the acute care and chronic care models of disease management. Although attempts are underway to change the delivery of care, our current system of COPD management for the hospitalized patient reflects the 'tyranny of the urgent' [82], with most clinical resources dealing the necessity of acute intervention for the very ill patient.

iv) The complex problems of the at-risk COPD patients, with severe disease, profound systemic effects, insufficient self-management skills, often-lacking lines of communication, and frequent, severe co-morbidity is best handed by an interdisciplinary team; this is often not present in routine management.

v) The emphasis of returning the discharged COPD patient back to the care of primary care professional can be a double-edged sword. Certainly, the primary care provider must be engaged to realize the full benefits of any comprehensive approach. However, in the peri-hospitalization period, the complex COPD patient with severe, unstable disease, often major changes in medical treatment, and with common co-morbid conditions (such as CHF) is best managed at least temporarily by the interdisciplinary team that is acquainted with this individual from the index hospitalization. Thus, proper transitioning from acute care to chronic care, incorporating and refining both approaches, is essential to optimal management of this patient.

vi) Current medical care for the complex patient with exacerbations of chronic disease such as the COPD patient is highly disjointed. In many cases the COPD patients cannot name their hospitalists. There is no "go-to" individual to call. The primary care physician is typically "out of the loop": for example, in the Jencks paper mentioned above [75], about one-half of the Medicare patients who were re-hospitalized within 30 days had apparently not seen their primary care provider over this interval. Furthermore, there is little communication among crucial health care providers managing patients over this very-critical post-discharge period. In our experience, even with electronic medical records, the primary care provider often does not know that his/her COPD patient had been even admitted to the hospital. Clearly, continuation of medical services is crucial during the vulnerable post-hospitalization period.

vii) Patients with COPD, despite aggressive attempts at collaborative self-management education from experienced professionals, often do not become successful self-managers, and it is these unsuccessful self-managers who are at particularly high risk for subsequent health care utilization [83]. 


\section{A very brief summary of previous studies of practice redesign for COPD}

Treating the complex patient with COPD, especially in the peri-hospitalization period is a challenge both to that patient's health care providers and to the system. It certainly involves much more than simply prescribing bronchodilators. Patients admitted with COPD exacerbations or who have had frequent exacerbations often have severe primary lung disease. Beyond treating the lung disease, including airflow limitation, hyperinflation, mucus hypersecretion and gas exchange abnormalities, consideration must be given to recognizing and treating its often prominent systemic consequences and frequent comorbidities. Systemic effects are increased during exacerbations, and frequent comorbidities also contribute to total disease burden $[84,85]$. As stated earlier, current care is often reactive, by necessity focusing on the problems associated with the exacerbation [86]. Even then, the pervasive nature of the exacerbation often requires an interdisciplinary approach that may not be available to the institution. Finally, for optimal care, proactive management is necessary to provide the preventative, educational, social services to optimize outcomes.The chronic care model $[82,87]$, which is now about 15 years old, was developed to address the needs patients with chronic illness. This model has six components: i) self management support; ii) clinical information systems; iii) delivery system redesign; iv) decision support (guidelines); v) health care organization; and vi) community resources $[82,87]$. These components are designed to tailor therapies to the individual patient and integrate services across settings. Most new comprehensive approaches to management of COPD have the chronic care model at their root.

A definition of integrated care from an American Thoracic Society workshop defined it as, "The continuum of patient centered services organized as a care delivery value chain for patients with chronic conditions with the goal of achieving the optimal daily functioning and health status for the individual patient and to achieve and maintain the individual's independence and functioning in the community" [88]. As a concept of care, integrated care is multidimensional, having system, organizational and clinical levels [89], making it somewhat confusing when specific aspects are not clearly delineated. Clinical integrated care for the COPD patient, which pertains to this discussion, is based on the chronic care model, with - arguably - additional emphasis on organizational and transitional care. Examples of clinical integrated care include a holistic approach, addressing the burdensome symptoms and disability from the disease, its systemic and comorbid conditions and from its therapy; management strategies over the entire trajectory of COPD, such as smoking cessation, promoting regular exercise and activity, optimizing pharmacotherapy, collaborative self-management, palliative therapy and hospice care; and (not to be overlooked) coordination of care among health care providers.

Other approaches to medical management have had elements of the entire package of clinical integrated care. Some examples include:

\section{Bundling of services}

Pre-discharge bundle of services at the time of the COPD discharge home includes, in different studies, a review of medications, educating on inhaler technique, assuring the patient has access to medications upon discharge, educating on medication adherence, and assuring the patient has prompt follow-up with health care providers. This type of intervention, although it makes great sense, has had limited success in reducing re-hospitalizations [90] - possibly because the items chosen for the particular bundle may not have been the ones needed in this situation, possibly because the positive signal from such an intervention may have not been sufficient to produce detectable change [91].

\section{Self-management}

Supported self-management in COPD includes efforts at educating the patient to become more efficient at practices that are involved in self-efficacy. This typically includes promotion of a healthy lifestyle (including smoking cessation, exercise, physical activity any proper nutrition), education on the early recognition and prompt treatment of the exacerbation, and better adherence to the often complex treatment regimen. This intervention has had mixed success when tested critically in randomized trials [92]. Part of this inconsistency may be due to the finding that only about $40 \%$ of patients with COPD when given self management education in selected trials actually become successful self-managers [83]. Adding to this is the under-emphasis of care coordination in some self management trials.

\section{Pulmonary rehabilitation}

Pulmonary rehabilitation, which includes self management education and exercise training, has demonstrated positive outcomes across several areas, including improvement in symptoms, exercise capacity and health status [36]. Additionally, when provided shortly after a hospitalization for an exacerbation, it appears (from a systematic review) to be related to reduced health care utilization, and even mortality [93]. This comprehensive intervention provides many of the components of integrated care, but does often fall short with respect to care coordination across settings.

\section{Health coaching}

A recently-described model of health intervention, named health coaching by the investigators, utilized motivational interviewing techniques, self management education, and limited care coordination. This intervention, which perhaps was incorrectly named since it incorporated more than health coaching, did produce impressive positive outcomes, including reduction in health care utilization, in the trial reported [94].

\section{Patient-Centered Medical Home}

This model of care was originally designed to reorient medical care back to the primary care provider. It includes the following components: 1) care across the course of the disease, prevention and wellness, acute care, and chronic care; 2) A holistic approach to medical management that families as well as the patient; 3 ) Coordination of care across the entire health care system,; and 5) Greater accessibility of services [95,96]. As a concept, therefore, this comes very close to the integrated care model.

\section{Delivery system design and decision support}

\section{Key points}

- The Chronic Care Model has become the paradigm for effective treatment of persons with chronic disease; this model has four components, self-management; delivery system redesign (eliminating silos of care); decision support (e.g., practice guidelines); and information systems

- Guideline development should have a multidisciplinary collaborative framework, with central coordination and be free of proprietary influence

- Unfortunately, clinical practice guidelines, as they currently exist, do not adequately address co-morbid conditions that are common to COPD

Delivery system design and decision support are two key components of integrated care (IC), also known as the chronic care model of disease management (CCM). he other two components are self-man- 
agement and clinical information systems [97]. In a systematic review of approaches to CCM, Adams and colleagues [98] noted that the clinical studies in which healthcare use was reduced had included at least two of these following four IC components:

1. Self-management techniques that increase the patients' ability to be the manager of their own condition.

2. Delivery system design to reorganize the clinical team to provide patients with prompt access to a knowledgeable health care provider

3. Decision support to enable clinical management to be guideline based and supported through provider education.

4. Information systems, to enable disease tracking, registries and rapid feedback to the point of care.

This article will comment on delivery system design and decision support.

\section{Delivery system design}

In many jurisdictions, healthcare is funded in silos in which primary and secondary care clinicians, hospitals and community resources operate in isolation from each other. Silo activities may function satisfactorily during episodic reactive acute care but do not optimize the ongoing needs of those with chronic complex conditions who require support across the spectrum of care at different times and from different locations. Not only is a silo system difficult for the patient to navigate but it enables medical error, under-diagnosis, inadequate monitoring of chronic conditions, loss of patient and caregiver preference, insufficient education to participate in self-management, omission and duplication of services [97]. In essence, it has been associated with poor quality care, high costs, low patient as well as provider satisfaction.

It is important to CCM system design that there be a clear understanding of the roles and tasks within a multidisciplinary healthcare team. Team functions and practice systems need to be reorganized such that appointments and follow-ups are scheduled to meet the needs of the chronically ill patients. Planned interactions to support care, clinical case management for complex patients and regular follow-up by the care team should include a focus on function as well as prevention of exacerbations and secondary complications. Care must also be understandable to the patient as well as being culturally appropriate. The entire system should be overseen by care networks or local advisory groups consisting of patients, healthcare providers and healthcare funding agencies. Many of the components of the CCM model, such as an action plan for exacerbations, patient education, self-management, supervised exercise training and psychological as well as social support are increasingly found in pulmonary rehabilitation programmes. In COPD, the clinical reach of the CCM model extends further, beyond these modalities to address issues of ongoing support that include long term follow-up and maintenance [99]. The extended rehabilitation capability may then function conceptually either as a bridge between the acute care hospital and the community, or as a buffer, accepting enrolment directly from the community with the intent of avoiding the reducing the requirement for hospitalization.

An early example of CCM was the guided care model [100], in which a registered nurse enrolled patients with COPD from the offices of several primary care physicians. The nurse carried a full assessment including medical, cognitive and functional status and then developed an evidence based care guide together with the primary care physician, which was entered into an electronic database. This guide was discussed with the patient and the caregiver and a simplified action plan was displayed at home. Those enrolled underwent a 15 hour lay led course in disease self-management. Patients were monitored with monthly calls and coached using motivational interviewing. Other functions included coordinating transitions between sites and providers of care, updating the electronic medical record, communicating with physicians, educating caregivers and assistance accessing community resources [100]. This model of care has been reported to result in a decrease in resource utilization and an increase in patient and provider satisfaction [101-105]. Several other models, described elsewhere, have used similar elements and have observed a positive impact on healthcare utilization, with fewer hospital admissions among the study group [106-108].

\section{Decision support}

Decision support involves embedding evidence based clinical practice guidelines (CPG) into daily practice. These should promptly available and shared with patients and their families to encourage participation. Provider education and integration of approaches to treatment among specialists, generalists and primary care, are also enabled through decision support. CPGs should be considered as part of clinical judgment. However, as stated in the National Clinical Guideline Centre (NICE) guidelines for COPD management, they should not override healthcare professionals making informed clinical judgments based in part on product characteristics of medications being considered [109]. CPGs often do not address multi-morbidity. Therefore, a busy practitioner attempting to implement several CPGs may have to balance increased risks of adverse drug interactions, patient preferences and the time imposition on the patient for monitoring [84]. A vision statement on guideline development for respiratory disease following an American Thoracic Society - European Respiratory Society workshop on guideline development [110] noted the need for a multidisciplinary collaborative framework, with central coordination, funded free of proprietary influence. It was suggested that the evidence be shared among an international committee of authors who could jointly review the evidence and the guideline recommendations. Questions answered in the guidelines should be those important to patients, clinicians and other stakeholders. Guideline development should include standardized metrics to grade the quality of the evidence and the recommendations. Committees infrequently address the challenge of co-morbidities or provide advice on how clinicians, patients and others might implement them. For CPG development to be successful, in addition to ensuring rigorous standards by assembling the most knowledgeable clinicians, there does need to be expertise in guideline methodology. The vision statement also encourages guideline development committees not to hesitate in considering new ideas, diverse perspectives and unique contributions.

In 2008, the New England Healthcare Institute reported on barriers and strategies that might influence physician adherence to CPG [111]. This publication noted the importance of evidence based CPG as the "rules of the road" for physicians. These guidelines are the key to improving quality, outcomes and cost effectiveness of healthcare. To address the gap between agreed standards of care and actual practice, four categories of barriers were noted; payment (in some healthcare jurisdictions the metrics of care include volumes rather than outcomes, so there is little financial incentive to change behaviour); information technology (insufficient information access at point of care limits support for clinical decision making); physician culture (physicians tend to rely on their own judgment and receive very little feedback on their process of decision making); and current development (lack of transparency in CPG development, lack of trust in the guideline, lack of guideline flexibility in clinical practice and failure to reflect complexity of practice). A number of recommendations were made as to practice changes that might enhance the use of CPG. The authors noted that guideline use varied very little with age ( $\leq 40$ years compared with $>40$ years) and that most physicians described themselves as "light" guideline users. 
In many jurisdictions, the healthcare system fails to meet the needs of patients with complex chronic conditions and multi-morbidity. System redesign is required to decrease silo care and provide prompt access to healthcare professionals across different locations at different time points in the disease. Practice guideline committees should be international and have standardized metrics. They need to address the issue of multiple chronic conditions as well as the variations in treatment responses. Guidelines should also provide the opportunity for modification based on local cultural, political and economic factors.

\section{Integrated care for COPD: patients versus systems}

\section{Key points}

- Clear terminology is needed in the area of integrated care for the COPD patient

- Good COPD care is multi component, multi professional and coordinated

- Patient engagement (and involvement) is critical

- Chronic care models seem to work

- Technology alone is not the solution

- Population level commissioning is the way forward

When the subject of integrated care for COPD is discussed, it is assumed that we are all agreed on a definition. In fact, this is not the case, and a review of the literature reveals many variations on what we mean by the term integrated care. The British Thoracic Society has recently published a statement on the subject and defines integrated care as "The best possible care for the patient, delivered by the most suitable health professional, at the optimal time, in the most suitable setting" [112]. While no one would disagree with this idealistic sentiment the definition does not explain how such an outcome could be delivered. In an attempt to clarify the situation it is possible to consider aspects such as the components of good care, the delivery of care through coordinated programmes and the commissioning of appropriate care pathways by payers.

The clinical guidelines available today recognize that COPD care requires a modular approach. The components include prevention (smoking cessation), drug therapy, rehabilitation, exacerbation planning, self-management training and - eventually - palliative care. In addition, most patients with COPD will have other co-morbid conditions that will require appropriate attention. While not every patient will require the same selection of treatment options, it is important that the individual patient has their need for each component assessed. These days it is very unlikely that a single health professional will be responsible for the total care of the patient; therefore, the delivery of care will require a multi-professional team as well as involvement of the patient. In the context of delivery, integrated care can be considered to be multi-professional care that crosses the traditional boundaries of primary and secondary care (vertical integration). Such an approach has been shown to reduce hospitalizations and improve symptoms. In some circumstances the integration may extend to social care and even local government.

It is desirable for the delivery of integrated care to be coordinated and de-fragmented. Ideally this should be by a single provider capable of providing all necessary aspects of care and involving the patient at the center of decision making. The most common theoretical model of care in this respect is the Chronic Care Model [97]. In this case, the knowledgeable dialogue between patient and health professional is supported by information technology and clinical guidelines as well as a structured delivery mechanism. Programmes that offer at least two components of the Chronic Care Model have also been shown to reduce hospitalizations [98]. There is some suggestion, though, that such comprehensive approaches have greater benefit to those patients most at risk from hospitalizations. The delivery of programmed care to those patients in primary care who are not at great risk from hospitalization may not have much to gain [113]. The position of digital solutions to care provision also remains uncertain. Tele-monitoring alone does not seem to offer any significant benefit when added to an existing effective programme [114]. In some parts of the world digital approaches to care delivery have been successful while in others this has not been the case.

We usually consider integrated care for COPD from the standpoint of provider structures. It is important to realize that COPD should be an area of interest to commissioners or payers. COPD imposes a substantial healthcare burden in terms of premature mortality and resource usage. It is associated with late inaccurate diagnosis and there are large variations in the quality of care. Commissioners therefore need to take an interest in developing clinical pathways that consider the whole population and also provide value for the tax payer. In many countries there is a barrier between primary care and access to a specialist.

From a patient's perspective, treatment by a specialist would always be preferable. On the other hand, for a system to deal with a common condition like COPD most clinical contact will have to take place in a primary care setting. For far too long we have tolerated a system where disease-specific expertise is locked behind a referral barrier which prevents access for many patients and transfer of knowledge and skills across the system. In effect, specialists may become a barrier to progress to improving access to expert care so a change in the model is required [115]. There are several ways that commissioners can help to improve the system by encouraging vertical integration. Firstly, specialists can enhance the skills of general practitioners and other primary care staff by taking part in multi-disciplinary meetings, webinars or virtual clinics. It is also possible to move away from the stereotype of hospital specialist and general practitioner towards a model where specialists work in and acknowledge their responsibility towards the community that they work in. In the UK, it is becoming increasingly common to appoint respiratory physicians with a responsibility for services in the community as well as their base hospital.

Traditionally, we think of commissioning services for COPD in terms of the needs of individual patient. This approach does not necessarily ensure that each patient necessarily ends up receiving the right care at the right time or place. Population level commissioning is an approach that can produce the right care for each patient and simultaneously provide the best value for the system. This approach requires the ability to stratify the population by risk or severity and also ensure that there is a sufficiently robust clinical commissioning pathway so that people are treated in the correct environment. Other specialties such as diabetes have become more experienced in such population approaches and use clear clinical pathways to identify where patient are best managed. It should be possible to do this easily with COPD. For example there are clearly some things like lung volume reduction assessment, respiratory failure and advanced disease that require the technical services that exist only in hospitals. Other services such as pulmonary rehabilitation, self-management training or spirometry can be undertaken in a community setting. Case finding, medicines management and influenza immunization can be conducted in the local surgery. Some things like smoking cessation need to be available in every domain. The commissioner's role is to design and broker solutions along these lines that suit the characteristics of the population. 
So far, we have not mentioned the patient's role in their care. One of the principles of the chronic care model approach is that the patient is not only at the center of care but also that they take an active and informed part in decisions about their care. One of the cornerstones of the political reforms of the health service in England earlier this decade was the mantra "no decision about me without me". For integrated care services to fulfill their potential the patient must be aware and informed about their condition and have the knowledge and confidence to demand the best from their health care professional team. Unfortunately, we are still some way from achieving this ideal.

\section{Self-management and health information technology}

\section{Key points}

- Self-management education aims a sustained, adaptive behaviour change in targeted areas

- Systematic reviews have shown positive outcomes for COPD patients undergoing self-management interventions

- Health information technology can improve lines of communication among patients and caregivers

\section{Introduction}

Many countries have recognized and endorsed the need for self-management education within national standards of care. The premise is that if patients receive effective support/coaching they can be empowered to adopt the behaviour needed to cope with their disease on a day-to-day basis. By doing so, medication adherence will be improved, exacerbations will be better managed, healthy behaviours (exercise/physical activity) will become second nature, and many of the poor outcomes related to chronic disease can be averted [116].

Systematic reviews have shown positive outcomes for COPD patients undergoing self-management interventions. A recent Cochrane review [117] demonstrated that self-management interventions led to a lower probability of respiratory-related hospitalization (odds ratio $0.57,95 \%$ CI 0.43 to 0.75 , nine studies, 1749 participants).There was also an effect of self-management interventions on improving health status (St. George's Respiratory Questionnaire total score, mean difference -3.51, 95\% CI -5.37 to -1.65, 10 studies, 1413 participants).

However, challenges remain understanding why some self-management interventions succeed while others fail. The answer to this question cannot be found by simply adding up the contents of successful interventions while subtracting the contents of the unsuccessful ones. After looking more closely at the trials, it becomes evident that programmes more likely to be effective have the following characteristics:

- Self-management education aimed at behaviour changes, targeting a patient's goal.

- Timely access to health care.

- Support/coaching by health care professional such as a case manager.

- Carefully considering disease severity and comorbidities.

\section{Self-management education intervention aiming at behavioural changes}

The findings from some studies suggest that successful self-managers (i.e., those patients who follow self-management training instructions) stand to benefit from the self-management education. In a study by Bucknall and colleagues [118], 42\% of patients with COPD given self-management training became successful self-managers; this subgroup did have a significant reduction in the primary endpoint of the study (hospital admissions). In a study by Bischoff and colleagues [119], 40\% of patients with COPD were adherent to their written action plan for the exacerbation, and, likewise, this subgroup improved with respect to a more rapid recovery of their respiratory symptoms.

There may be many reasons to explain the low rates of COPD patients becoming successful self-managers. It is noteworthy that the majority of patients $(>50 \%)$ in the studies had only one exacerbation during the follow-up period - providing too few opportunities to implement the self-management skills taught during the initial intervention. Another reason could be a failure to intervene: the self-management training intervention was not properly constructed or implemented to change self-management behaviour or outcomes. An example of this would be in the negative trial by Fan and colleagues [120]. In this multi-center study, the self-management educational intervention was implemented according to protocol, in that $87 \%$ of patients in the intervention group completed all four individual self-management education sessions; and case managers covered $77-89 \%$ of the educational items and follow-up telephone contacts. However, a closer look at how the intervention was received by patients reveals that they failed to use the self-management skills that they were taught: they didn't initiate use of antibiotics and/or prednisone any earlier than those in the usual care group. Furthermore, although patients were instructed to call their case manager if they experienced an exacerbation that required medication, calls were made in only $4.5 \%$ of patients in the intervention group. In contrast, case managers in a positive trial of self-management by Bourbeau et al. [106] were contacted by patients in $48 \%$ of exacerbations.

In order to be successful, a self-management intervention has to lead to behaviour change in those behaviours targeted by the intervention. Most important, it is the process of supporting self-management, which refers to the strategies, techniques and skills used by healthcare providers (typically case managers) to instrument patients with the knowledge, motivation, confidence and skills required to effectively self-manage their disease.

Another important problem has recently emerged: There is concern that the health benefits from such self-management programmes in COPD could be counterbalanced by an increased mortality signal from the results of one study in particular [120].

It is clear that self-management should be carefully targeting disease severity and comorbidities. We have to look to find potential solutions to improve self-management intervention to increase patient adherence with respect to self-management skil//behaviors, to insure timely access to the health care professional and physician and to better target disease severity that it is done safely.

\section{Health information technology}

Health information technology is increasingly seen as a key tool for improving the quality and efficiency of care. It includes electronic health records; personal health records; use of personal digital assistants; health information exchange; computerized order entry systems; e-prescribing; and disease-specific or population-based registries.

Health care systems are counting on health information technology to facilitate the implementation of chronic disease management programmes, thereby reducing preventable hospitalizations and adverse events. Specific to COPD, data on the utility on health information technology to the management of this chronic respiratory disease are limited and suffer from several problems, including small patient numbers, variable intensities of monitoring, and the lack of pre-determined 
treatment plans. Recently, the American College of Chest Physicians and the Canadian Thoracic Society guideline on Prevention of Acute Exacerbations of COPD concluded that, "telehealth compared to usual care does not result in prevention of acute exacerbations (ER, hospital admissions) over 12 months" [121]. Thus, clearly more information on indication, implementation and setting is needed to make this intervention successful. Health information technology has been long on promises and short on details. We need more properly designed studies.

Despite the limited support from the existing evidences in COPD management, health information technology is expected to play a critical role in improving respiratory chronic disease outcomes. There are several potential roles of health information technology:

- Providing personalized support integrated care in chronic disease management.

- Enabling decision-support for personalized evidence-based treatment.

- Permitting chronic disease registries to be created to identify, monitor (outcomes and practices), target at risk populations.

Health information technology can be simple or complex. Simple systems include clinical intervention with single feature, such as automated telephone contact, email communication, or website or videoconference access. Complex systems include clinical interventions that have more than one single component, such as combinations of email communication, videoconference, web site, and telehealth.

\section{Providing personalized support integrated care in chronic disease management}

The COPD Patient Management European Trial (COMET) provides an example of how it might be possible to use health information technology to support a self-management education intervention [122]. This trial includes 345 patients from 33 centers in 4 European countries. The objective of this pragmatic trial was to evaluate a multi-component home-based COPD self-management programme, Living Well with COPD (translated in 4 languages and adapted for the trial), compared to usual care on all-causes hospital days in severe COPD patients over 1 year follow-up. The programme involves self-management education and health coaching by case managers. Beyond its implementation across culturally-diverse countries with distinct health care systems, the combination of its self-management educational programme with an e-health platform is novel. Key components of the e-platform are: 1) Weekly/daily reporting by patient with automatic analysis of clinical symptoms through an electronic data system; 2) A dedicated vocal server to early detect and treat exacerbations; and 3) Worsening of clinical status triggers an alarm, leading to a standardised nurse intervention and transmission to the referent hospital physician for a medical decision. Another methodological aspect of the study is the attention implementing quality assurance with respect to the regular training of the health care professional case managers, standardization of the educational programme, and support by the case managers during the study. Furthermore, this study does not limit assessment to only trial outcome measures such as patient health and hospitalizations, but also includes process measures (e.g., patient acquisition of skills and behavioural changes). The intent is to gather information that could facilitate the interpretation of trial outcomes that are still to come.

\section{Chronic disease registries}

Health information technology could also permit chronic disease registries. An example is a standardized, web-based registry for chronic disease has been developed and implemented in the primary care practice in Montreal, Quebec, designed to assist health care professionals in treating COPD patients. The registry includes different components: patient, inscriptions, diagnostic, quality indicators, medication and lab. The following specific indicators of are monitored in the practice:

- Physician (practices): Written action plan and access to prescription with communication of health care professional (case manager).

- Patient (outcomes): Specific behaviours ( $\leq 3$ days recognition of the exacerbation followed by appropriate actions; health-related quality of life.

- Health care system (outcomes): Primary care provider unscheduled visits, emergency department visits, hospital admissions.

Implementation of this registry has made it possible to identify those patients who had difficulty in managing COPD exacerbations. Implementing a programme with an interdisciplinary approach (involving a nurse and/or a respiratory therapist with the family physician) could then bridge this gap. Data are collected on specific aspects of the management of exacerbations, including its early recognition and treatment and actions taken to prevent emergency department visits and hospital admissions.

In the year before implementing the programme only $10 \%$ of patients with exacerbations had a written action plan, leading to delays in consultations and treatment. After the implementation of the programme, most of the patients with a history of exacerbations had both a written action plan, and a nurse or a respiratory therapist to coach them in its implementation Two-thirds of the patients at 3 and 6 months reached pre-defined quality indicators of adopting the proper behaviour in selfmanaging exacerbations; this included following the action within 3 days of symptom worsening and adjusting action (calling the case manager) if not improving within 2 days.

These examples suggest that health information technology has a potentially important role in improving outcome in COPD. However, in order to make progress, health information technology has to be welldefined with respect to aims, process and outcomes. Its rationale must be more than simply good intention, and well-designed studies are needed to provide more useful data in this area.

\section{Integrated care for patients with COPD in Italy}

\section{Key points}

- The estimated prevalence of COPD in Italy ranges from $4.5 \%$ (general population) to $6 \%$ (age 46 and 55 years) to $11 \%$ (those over 55 years old)

- The need to improve care of COPD through integrating services has led to several projects in Italy; several research projects are still in progress

- Greater accessibility to pulmonary rehabilitation, greater emphasis on education, and telemedicine assistance are seen as important components of integrated care

The estimated prevalence of COPD in Italy ranges from $4.5 \%$ of the general population, up to $6 \%$ of individuals aged between 46 and 55 years, and $11 \%$ of those over age 55 [123]. Differences in reported prevalence rates depend on diagnostic criteria and regional differences, with higher prevalence rate in males and ex-smokers [124]. COPD is a risk factor for several comorbid conditions, such as heart disease, and non-psychotic mental disorders, included depression, with higher impact in elderly patients [125]. Several variables are 
identified as factors influencing COPD life expectancy, including smoking, severity of dyspnoea, age, exercise capacity, body mass index (BMI), exacerbation rate, comorbidities, and quality of life. Severity of COPD (as assessed by Global Initiative for Chronic Obstructive Lung Disease [GOLD] stage), comorbidities and low BMI may also predict mortality or hospitalization risk in an Italian population [126].

Administrative data derived from hospital admissions and pharmaceutical prescriptions may lead to underestimation of COPD prevalence or incorrect identification, because patients suffering from COPD usually receive diagnosis and treatment as outpatients, and hospital admissions predominantly occur only in the most severe stages. Epidemiological data from general practitioners (GPs) data bases showed good identification of COPD subjects and consistency with prevalence data from National Institute of Statistics (ISTAT) surveys [127]. A recent Italian study reported a 3200 average year cost to manage COPD patients, with a $20 \%$ increase compared to a study conducted in a similar population in 2008 $[128,129]$. Direct costs were mainly represented by hospitalizations (67.2-70.7\%), and occurrence and type of exacerbations were the main drivers of direct health care cost. In a secondary data analysis of healthcare administrative databases of the Lombardy region, two and half years after a hospitalization health care resource consumption was about $6700 €$, along with an increased risk of relapse of exacerbation and mortality [130].

In Italy, the Chronic Care Model has been widely implemented in primary care since 2009 . National and regional policies were until now addressed to the definition of integrated diagnostic and therapeutic pathways of care for several chronic conditions (including COPD) through collaboration among hospital and community services [131]. Even if an increase of economic resources consumed for outpatients was observed in the recent years, further changes are claimed to increase the capability of territorial management of moderate-to-severe COPD patients [128,132,133].

Adequacy of drug prescription, promotion of patient's treatment adherence, pulmonary rehabilitation, and individualized long-term treatment strategies are proposed as interventions to decrease the rate and severity of exacerbations of COPD [132]. The prevention and prompt treatment of COPD exacerbations should include the reduction of risk factors (smoking cessation, vaccination), inclusion in programmes of respiratory rehabilitation, and promotion of self-management strategies. Collaboration of hospital and community services aimed to the health maintenance and prevention of hospital admissions is proposed in the National Chronicity Plan being developed by Italian Ministry of Health [134]. Integrated diagnostic and therapeutic pathways of care, intermediate levels of care between hospital and primary care and pulmonary rehabilitation are among the objectives to ensure that the patient be diagnosed and managed in the community and referred to the specialist when appropriate.

However, there are differences among regions, and some other criticisms still hinder the widespread application of integrated care these patients. In an observational study of patients with COPD diagnosis conducted by Italian general practitioners, sufficient control of the disease was reported only in $47.2 \%$ of the subjects with over two exacerbations in the last 12 months [135]. Suboptimal adherence of GPs practice to the guidelines for COPD diagnosis and management was shown in a study conducted in Lombardy region, and confirmed in studies conducted in other regions [135]. These studies highlighted the need to improve the knowledge of mechanisms of COPD progression among GPs, promoting the use of spirometry for the diagnostic definition, and the prescription of pulmonary rehabilitation to symptomatic patients [136].
Some organizational issues may limit the accessibility to pulmonary rehabilitation for Italian patients with chronic disabling respiratory diseases. Compared to the actual prevalence of COPD patients in Italy (2.5 to 3 million), in a national study published in 2004, only 53 rehabilitation departments and/or pulmonology units were estimated to warrant availability and accessibility to pulmonary rehabilitation, with more than half of them being located in the north of the country [137]. In some regions, the differences in availability of pulmonary rehabilitation and integration between acute hospital and primary care may be related to the higher occurrence of potentially avoidable hospitalization for COPD [138,139]. Accessibility to pulmonary rehabilitation may be also hampered by differences among regional application rules of national health policies across Italy, that frequently made delivery of pulmonary rehabilitation in unfavourable competition with rehabilitation of musculoskeletal and neurological conditions [140].

Different models of integrated care programmes for COPD patients were developed in recent years, and ongoing projects will undoubtedly help determine the feasibility of different components, including the application of information and communication technologies (such as telemedicine) to self-management and pulmonary rehabilitation [141].

Patient education programmes are widely included in pulmonary rehabilitation programmes, and showed effective in improving patient knowledge and self-management [142]. A multicentre randomized controlled trial involving 4 European countries - including Italy - was recently approved to evaluate effectiveness of a home-based COPD selfmanagement programme to reduce exacerbations and hospital admissions. The self-management programme will include extensive patient coaching by healthcare professionals to improve disease management skills, an e-health platform for reporting health status updates, and elaboration of a clinical score for early detection of clinical instability (COMET study) [122].

Home based tele-management of COPD patients in remote areas of the Lombardy region of Italy has been performed providing home-based tele-management services for patients with chronic heart failure and COPD, as well as second-opinion tele-consultations in cardiology, dermatology, diabetology, and pulmonology for general practitioners [143]. A recent retrospective study exploring patterns of usage of home-based tele-management in COPD, showed that "frequent relapsers" are most likely to require scheduled and unscheduled telemanagement services [144]. Additionally, in a recent retrospective study of chronically hypercapnic COPD patients on long term oxygen therapy, tele-assistance combined with non invasive ventilation reduced the frequency of exacerbations [145]. In contrast, a crossover randomised controlled trial showed that telemonitoring added to standard care did not improve either the time to next acute hospitalization, or health-related quality of life in COPD [146].

An ongoing research project in the Tuscany region of Italy, funded by the National Health Ministry Research Grant, is currently exploring cost-effectiveness of the implementation of a system of telecare in COPD patients with multiple morbidities. This system uses simple, low-cost tools (symptoms diaries, action plans) and frequent contacts with health personnel. Preliminary data show an increase of knowledge level of GPs in COPD guidelines adherence, with high rate of treatment modification (53\%) after enrolment in the integrated care programme (unpublished data).

In summary, the optimal care for patients with COPD requires a patient-centred approach, integrating medical care among healthcare professionals redesigning hospital and home care pathways. This need has led to the development of novel approaches in Italy. This is currently a work in progress. 


\section{The CIRO+ Program: an integrated, holistic, personalized medical strategy}

\section{Key points}

- A thorough patient assessment is mandatory at the beginning of every rehabilitation program

- Socio-technical systems theory can be very supportive for organisation of pulmonary rehabilitation

- Outcomes after pulmonary rehabilitation are non-linear, differential and multidimensional

- Pulmonary rehabilitation improves health through improving the patient's well being

Cardiovascular diseases, cancers, chronic respiratory diseases and diabetes are the four major chronic non-communicable diseases (NCDs). To a great extent, NCDs develop as a result of an unhealthy lifestyle, such as tobacco use, physical inactivity, unhealthy diet or the excessive use of alcohol [147]. The risk factors for the major NCDs are still increasing worldwide. One of the greatest challenges to healthcare management is to understand the growing complexity of these chronic NCDs. Current health care ignores this heterogeneity in the burden of NCDs and largely fails to offer a personalized, patient-centred approach to management.

It is recognized that COPD is a complex, heterogeneous syndrome with numerous pulmonary and extra-pulmonary components [148]. The presence and severity of COPD has been traditionally diagnosed by assessment of the degree of airflow limitation. However, it is nowadays generally accepted that accurate characterization can not be achieved by isolated use of measures of airflow limitation such as the $\mathrm{FEV}_{1}$ [148]. Significant heterogeneity exists in clinical presentation, physiology, imaging, response to therapy, decline in lung function, and survival amongst patients with COPD, irrespective of the degree of airflow limitation [148]. Furthermore, exacerbations and comorbidities contribute to the overall disease severity in individual patients [149].

Recognizing the importance of factors other than airlow limitation, the Global Strategy for the Diagnosis, Management and Prevention of COPD (GOLD) has proposed a three-domain assessment of COPD, which includes the level of symptoms experienced by the patient and the risk of exacerbations [149]. It is now widely recognized that grouping of COPD into clinically-meaningful subgroups or phenotypes is needed to guide more effective therapies and management strategies. A COPD phenotype should be able to classify patients into distinct subgroups possessing uniform and distinct features, with the aim of facilitating the assessment of biological mechanisms, prognosis and clinically meaningful outcomes [148]. Furthermore, sub-grouping of patients may be helpful to identify those who stand to benefit from a particular treatment, such as pulmonary rehabilitation.

A patient-centred approach of patients with chronic respiratory diseases such as COPD is not new in pulmonary medicine. Indeed, the first authoritative statement of pulmonary rehabilitation from the American College of Chest Physicians, published in 1974, introduced pulmonary rehabilitation already as an "art of medical practice", wherein an individually tailored, multidisciplinary programme was formulated [150]. Through accurate diagnosis, therapy, emotional support and education, pulmonary rehabilitation stabilizes or reverses both physiopathological and psychopathological manifestations of pulmonary diseases. Also, it helps return the patient to the highest possible functional capacity allowed by the handicap and overall life situation [151].

In 1994, the National Institutes of Health defined pulmonary rehabilitation as a multidimensional continuum of services for the patient and the family supplied by an integrated team of specialists in comple- mentary disciplines, with the goal of the patient living and functioning independent within society [152]. The 2013, the American Thoracic Society - European Respiratory Society Statement defined pulmonary rehabilitation as, “... a comprehensive intervention based on thorough assessment followed by patient-tailored therapies designed to improve the physical and psychological condition of people with chronic respiratory disease and to promote the long-term adherence to health-enhancing behaviors" [36]. The latter part of this definition fits with the definition provided in the addendum in the American Association for Respiratory Care, which stipulates that pulmonary rehabilitation should be both restorative and preventive [153].

The major, common points among the various definitions of pulmonary rehabilitation include: i) a focus on chronic respiratory patients and their care givers; ii) an individualization of the intervention; iii) an ongoing multidisciplinary intervention; iv) outcomes based on physiological, psychological and social measures considering a global dimension to the individual's health; and v) the stimulation of longterm adherence to health-enhancing behaviours in order to promote autonomy and social participation of the patient.

The diagnosis of physiopathological and psychopathological problems in the individual patient forms the start of every pulmonary rehabilitation programme. Such assessment is performed in every referred patient for pulmonary rehabilitation in the expertise centre, CIRO+. This centre for integrated rehabilitation for chronic respiratory conditions is located in the southern part of the Netherlands. CIR0+ is a member of the Dutch Lung Centers, specialised third-line centres for chronic respiratory care.

Pulmonary rehabilitation advocates a personalized approach, and aims for patients with COPD to acquire and apply participatory and preventive skills to make them, together with healthcare professionals, more pro-active in the daily management of their disease. This process of healthcare organization around the patient (i.e., adopting a patientcentered approach) is generally underestimated or neglected in the management of patients with chronic conditions as COPD [154].

Ideally, COPD management must offer a flexible, holistic, and integrated intervention, based on partnering of different skills to achieve shared, individualized, patient-related objectives, with a goal to achieve improvement in clinically-relevant outcomes and added value to the patient and the community. This requires a process-based organization to manage business around these core processes, including intake and assessment, rehabilitative therapies, and outcome evaluation [155].

Socio-technical systems theory has the following characteristics: i) designing and changing organizations in response to environmental conditions and strategic choices; ii) addressing the increasing complexity of organizations that results from increasing external uncertainty and variation within the internal division of labour; and iii) offering a tailor-made, individualized programme [156]. Socio-technical theory offers a framework to improve efficiency, quality, flexibility and innovation. Indeed, a key feature of socio-technical design involves bringing together people from different roles and disciplinary backgrounds who have different skills, experience and expertise. Pluralism is the norm, and this implies they share their views and expertise. Participants from different backgrounds educate one another in the opportunities that may exist for the design of a new system, each contributing to the design process [157]. Socio-technical theory offers a framework for healthcare organizations to create value through improving outcomes that matter most to patients, always taking into consideration the costs of achieving those outcomes [158].

CIR0+ rehabilitation centre is based on this socio-technical systems theory. Its approach is holistic and organized around the individual and his/her experienced needs. CIRO+ operates as an integrated practice unit (IPU) that treats not only a disease but also the related conditions, complications, and circumstances that commonly occur along with it. 
In an IPU, personnel regularly work together as a team towards the common goal of maximizing the patient's overall outcomes as efficiently as possible. Actually, organizing pulmonary rehabilitation according to the socio-technical principles as set-up in CIR0+, meets the features of a high-value healthcare organization [159].

The pulmonary rehabilitation programme has a modular structure, with basic modules and step-up modules. Decision-making in step-up modules is based on the outcomes of the assessment procedure. Every module integrates a spectrum of activities in that particular domain. This modular approach makes it possible to individualize the intervention. Examples of treatment modules are physical fitness, hydrotherapy, individual outdoor activity, addressing body composition abnormalities, addressing co-morbidities, dyspnea management, exacerbation management, increasing life activity, functional training, adherence training, partner therapy, and physical fitness.

Based on experience with the CIR0+ programme, it became clear that responses after pulmonary rehabilitation are differential and multi-dimensional among patients. Furthermore, these responses are non-linear, so and clusters of very good and good responders could be identified. In that way, pulmonary rehabilitation can importantly downsize the experienced disease burden and can improve physical, emotional and social functioning of referred patients [160]. Indeed, pulmonary rehabilitation importantly supports patients to adapt and manage their own wellbeing, in light of the physical, emotional and social challenges of life [160].

Pulmonary rehabilitation can achieve these improvements in health status by considering patients as complex adaptive systems where illness is the result from complex, dynamic and unique interactions between the different components of the overall system. Because of this, outcomes are largely unpredictable and highly-dependent on the context in which the program is delivered [161,162], underscoring the need for all healthcare professionals to partner with the patient and work closely with other providers to enhance outcomes [154].

To summarize, CIRO+ aims to attenuate the personal and societal burden of chronic respiratory diseases through developing and implementing personalized, innovative diagnostic, preventive and therapeutic solutions and delivery systems.

\section{Medical management of the at-risk COPD patient}

For the purposes of this discussion, the at-risk COPD patient is either hospitalized or recently discharged following an exacerbation of the disease. The best model for treating the at-risk COPD patient has yet to be determined. The following is one proposal, based on an integrated care model for COPD management [88], focused on the high-risk patient, and enhancing lines of communication to provide a seamless transition of care from the hospital to the home and on into the community. Elements include standardizing education based on collaborative self-management, employing an interdisciplinary team intervention during and in the critical weeks after a COPD hospitalization and fostering lines of communication using a novel multi-directional telemonitoring system. This plan of care is specific to one institutional setting in one health care system, so - even if it works - its generalizability is open to question.

i) Initial focus on the hospitalized, dual-eligible COPD patient. This group of patients has markedly impaired health status and very high risk for subsequent health care utilization and mortality.

ii) Use of an interdisciplinary, COPD management team approach, including an advanced practice registered nurse (APRN), pulmonologists, hospitalists, respiratory therapists, pulmonary rehabilitation coordinators, a case manager managing telemonitoring, hospital case managers, hospital educators, a database coordinator, and administrative support staff. All these team members are currently hospital staff managing COPD as part of their duties. This team will meet quarterly.

iii) Standardization of COPD educational material for patients.

iv) Training of the team in self-management principles [163] and health coaching using motivational interviewing [94].

v) Decision support: standardized management plans for the hospitalized COPD patient, with attention to medications and discharge planning.

vi) Every hospitalized (therefore, at-risk) patient is evaluated within the first two days by the APRN, and subsequently by selected members of the interdisciplinary team, as needed, during the hospitalization. The APRN will see the patient throughout the hospital stay and (along with members of the team) within one week of hospital discharge. This ongoing interaction beginning early on in the hospitalization and continuing through that hospitalization and into the post-discharge period will foster the continuity of care that is often lacking. The APRN will serve as a liaison between the hospitalists, floor nurses, case managers, and subspecialist physicians. Furthermore, the APRN will also serve as the liaison to the primary care providers, home care professionals and selected professionals.

vii) Follow-up visit(s) to the team beginning within one week of hospital discharge. Despite comprehensive written and personalized instructions at the time of hospital discharge, discharge instructions are uncommonly followed exactly by patients, leading to pronounced confusion over medications, inhaler use, and follow-up visits. These follow-up visits will be used to clarify medication issues and to evaluate and treat destabilization of the disease process or worsening of co-morbid conditions such as heart failure. Patients will be contacted by the coordinator, and provided transportation, if necessary.

viii) Multi-directional telemedicine will be employed, enhancing communication in patient-caregiver, caregiver-patient, and caregivercaregiver areas, making it truly multidirectional in scope. All atrisk patients will be offered telemonitoring; this will be introduced to the patient at the time of the hospitalization, and implemented either during the hospitalization or during the first follow-up visit within one week of hospital discharge. Reinforcement of its use will be given during the one week hospitalization.

ix) The patient with dual morbidity - COPD and heart failure - will be managed using additional input from our hospital's CHF management team and utilizing a novel telemedicine program created to follow patients with both conditions.

x) Transitioning care of the COPD patient to the primary care provider is an integral component of the chronic care model and of this proposed program. During the initial, post-discharge, critical period where the interdisciplinary team is playing a major role in patient management, the primary care provider will have access to patient management data through the medical record and from evaluation notes generated by the team and sent directly to the provider. The team, by protocol, will notify the provider of the patient's recent hospitalization, and provide information on the assessments and changes in therapy. Following stabilization, the team will transition care to the health care provider; this will include updating diagnoses, treatments, special needs, and other interventions and sending this information to the provider. The goal is to return total management of a stabilized patient back to the provider. The team, however, will continue to be a resource to the primary care provider and could intervene again, when necessary.

xi) Quality improvement will be accomplished through regular, quarterly meetings of the COPD management team, and through monthly meetings of a team analysing reasons for hospital re-admissions in those patients re-admitted within 90 days; the latter subcommittee will report back to the total COPD management team. 


\section{References}

1. Proceedings of European Seminars in Respiratory Medicine Update 1998 (June 27-July 2, 1998). Monaldi Arch Chest Dis 53: 603-719.

2. Proceedings of the European Seminars in Respiratory Medicine Update 1999 (Moritz, Switzerland, July 2-6, 1999). Monaldi Arch Chest Dis 54:463-558.

3. Gibson GJ, et al. Respiratory health and disease in Europe: the new European Lung White Book. Eur Respir J 2013;42:559-63.

4. Knapp RG, Miller MC. Clinical epidemiology and biostatistics. Williams and Wilkins, Baltimore, 1992.

5. Sanders BS. Measuring community health levels. Am J Public Health Nations Health 1964;54:1063-70.

6. Viegi G, et al. Prevalence of airways obstruction in a general population: European Respiratory Society vs American Thoracic Society definition. Chest 2000;117(5 Suppl 2):339S-45S.

7. Halbert RJ, et al. Global burden of COPD: systematic review and meta-analysis. Eur Respir J 2006;28:523-32.

8. Celli BR, et al. An official American Thoracic Society/European Respiratory Society statement: research questions in COPD. Eur Respir Rev 2015;24:159-72.

9. Quanjer PH, et al. COPD (confusion over proper diagnosis) in the zone of maximum uncertainty. Eur Respir J 2015;46:1523-4.

10. Adeloye D, et al. Global and regional estimates of COPD prevalence: Systematic review and meta-analysis. J Glob Health 2015;5:020415.

11. Luoto JA, et al. Incidence of airflow limitation in subjects 65-100 years of age. Eur Respir J 2016;47:461-72.

12. Terzikhan N, et al. Prevalence and incidence of COPD in smokers and non-smokers: the Rotterdam Study. Eur J Epidemiol 2016;31:785-92.

13. Viegi G, et al. Prevalence rates of respiratory symptoms and diseases in general population samples of North and Central Italy. Int J Tuberc Lung Dis 1999;3:1034-42.

14. Maio S, et al. Respiratory symptoms/diseases prevalence is still increasing: a 25-yr population study. Respir Med 2016;110:58-65.

15. de Marco R, et al. Trends in the prevalence of asthma and allergic rhinitis in Italy between 1991 and 2010. Eur Respir J 2012;39:883-92.

16. Ekerljung $\mathrm{L}$, et al. Has the increase in the prevalence of asthma and respiratory symptoms reached a plateau in Stockholm, Sweden? Int J Tuberc Lung Dis 2010;14:764-71.

17. Bjerg A, et al. Increased prevalence of symptoms of rhinitis but not of asthma between 1990 and 2008 in Swedish adults: comparisons of the ECRHS and GA(2)LEN surveys. PLoS One 2011;6:e16082.

18. Backman H, et al. Decreased prevalence of moderate to severe COPD over 15 years in northern Sweden. Respir Med 2016;114: 103-10.

19. Nuvolone D, et al. Geographical information system and environmental epidemiology: a cross-sectional spatial analysis of the effects of traffic-related air pollution on population respiratory health. Environ Health 2011;10:12.

20. Maio S, et al. Urban residence is associated with bronchial hyperresponsiveness in Italian general population samples. Chest 2009; 135:434-41.

21. Eisner MD, et al. An official American Thoracic Society public policy statement: Novel risk factors and the global burden of chronic obstructive pulmonary disease. Am J Respir Crit Care Med 2010;182:693-718.

22. Celli BR, et al. Standards for the diagnosis and treatment of patients with COPD: a summary of the ATS/ERS position paper. Eur Respir J 2004;23:932-46.
23. Rabe KF, et al. Global strategy for the diagnosis, management, and prevention of chronic obstructive pulmonary disease: GOLD executive summary. Am J Respir Crit Care Med 2007;176:532-55.

24. Gulsvik A. The global burden and impact of chronic obstructive pulmonary disease worldwide. Monaldi Arch Chest Dis 2001;56:261-4.

25. Soriano JB, et al. Chronic obstructive pulmonary disease overview: epidemiology, risk factors, and clinical presentation. Proc Am Thorac Soc 2011;8:363-7.

26. Buist AS, et al. International variation in the prevalence of COPD (the BOLD Study): a population-based prevalence study. Lancet 2007;370:741-50.

27. Silverman EK, et al. Genetics and genomics of chronic obstructive pulmonary disease. Proc Am Thorac Soc 2009;6:539-42.

28. Viegi G, et al. Epidemiology of chronic obstructive pulmonary disease: health effects of air pollution. Respirology 2006;11:523-32.

29. Balmes J, et al. American Thoracic Society Statement: Occupational contribution to the burden of airway disease. Am J Respir Crit Care Med 2003;167:787-97.

30. Lundback B, et al. Not 15 but 50\% of smokers develop COPD? Report from the Obstructive Lung Disease in Northern Sweden Studies. Respir Med 2003;97:115-22.

31. Celli BR, et al. The body-mass index, airflow obstruction, dyspnea, and exercise capacity index in chronic obstructive pulmonary disease. N Engl J Med 2004;350:1005-12.

32. Cranston JM, et al. Domiciliary oxygen for chronic obstructive pulmonary disease. Cochrane Database Syst Rev 2005;CD001744.

33. Ram FS, et al. Non-invasive positive pressure ventilation for treatment of respiratory failure due to exacerbations of chronic obstructive pulmonary disease. Cochrane Database Syst Rev 2004:CD004104.

34. Schembri S, et al. Influenza but not pneumococcal vaccination protects against all-cause mortality in patients with COPD. Thorax 2009;64:567-72.

35. Alfageme I, et al. Clinical efficacy of anti-pneumococcal vaccination in patients with COPD. Thorax 2006;61:189-95.

36. Spruit MA, et al. An official American thoracic society/european respiratory society statement: key concepts and advances in pulmonary rehabilitation. Am J Respir Crit Care Med 2013;188:e13-64.

37. Jayes L, et al. SmokeHaz: Systematic reviews and meta-analyses of the effects of smoking on respiratory health. Chest 2016;150: 164-79.

38. Morris PB, et al, Cardiovascular effects of exposure to cigarette smoke and electronic cigarettes: clinical perspectives from the Prevention of Cardiovascular Disease Section Leadership Council and Early Career Councils of the American College of Cardiology. J Am Coll Cardiol 2015;66:1378-91.

39. Damarla M, et al. Discrepancy in the use of confirmatory tests in patients hospitalized with the diagnosis of chronic obstructive pulmonary disease or congestive heart failure. Respir Care 2006; 51:1120-4.

40. Pellegrin, R. et al. Interpretative strategies for lung function tests. Eur Respir J 2005;26:948-68.

41. Siafakas NM, et al. Optimal assessment and management of chronic obstructive pulmonary disease (COPD). The European Respiratory Society Task Force. Eur Respir J 1995;8:1398-420.

42. Falaschetti E, et al. Prediction equations for normal and low lung function from the Health Survey for England. Eur Respir J 2004; 23:456-63.

43. Lange $\mathrm{P}$, et al. Prediction of the clinical course of chronic obstructive pulmonary disease, using the new GOLD classification: a study of the general population. Am J Respir Crit Care Med 2012;186: 975-81. 
44. Black-Shinn JL, et al. Cardiovascular disease is associated with COPD severity and reduced functional status and quality of life. COPD 2014;11:546-51.

45. Rutten FH, et al. Heart failure and chronic obstructive pulmonary disease: An ignored combination? Eur J Heart Fail 2006;8:706-11.

46. Barr RG, et al. Percent emphysema, airflow obstruction, and impaired left ventricular filling. N Engl J Med 2010;362:217-27.

47. Smith BM, et al. Impaired left ventricular filling in COPD and emphysema: is it the heart or the lungs? The Multi-Ethnic Study of Atherosclerosis COPD Study. Chest 2013;144:1143-51.

48. Stone IS, et al. Lung deflation and cardiovascular structure and function in chronic obstructive pulmonary disease. A randomized controlled trial. Am J Respir Crit Care Med 2016;193:717-26.

49. Faludi R, et al. Diastolic dysfunction is a contributing factor to exercise intolerance in COPD. COPD 2016;13:345-51.

50. Agostoni P, et al. Exercise hyperpnea in chronic heart failure: relationships to lung stiffness and expiratory flow limitation. J Appl Physiol (1985) 2002;92:1409-16.

51. Torchio R, et al. Closing capacity and gas exchange in chronic heart failure. Chest 2006;29:1330-6.

52. Gargiulo P. et al. A non invasive estimate of dead space ventilation from exercise measurements. PLoS One 2014;9:e87395.

53. Apostolo A, et al. Impact of chronic obstructive pulmonary disease on exercise ventilatory efficiency in heart failure. Int $\mathrm{J}$ Cardiol 2015;189:134-40

54. Neder JA, et al, Exercise ventilatory inefficiency in mild to endstage COPD. Eur Respir J 2015;45:377-87.

55. 50liveira MF, et al. Heart failure impairs muscle blood flow and endurance exercise tolerance in COPD. COPD 2016;13:407-15.

56. Oliveira MF, et al. Effects of heart failure on cerebral blood flow in COPD: rest and exercise. Respir Physiol Neurobiol 2016;21:41-8.

57. Staszewsky L, et al. Outcomes in patients hospitalized for heart failure and chronic obstructive pulmonary disease: differences in clinical profile and treatment between 2002 and 2009. Eur J Heart Fail 2016:18:840-8.

58. WHO. A European framework to promote physical activity for health. 2007. Available from: www.euro.who.int

59. Pitta F, et al. Characteristics of physical activities in daily life in chronic obstructive pulmonary disease. Am J Respir Crit Care Med 2005;171:972-7.

60. Watz H, et al. Physical activity in patients with COPD. Eur Respir J 2009;33:262-72.

61. Waschki B, et al. Physical activity monitoring in COPD: Compliance and associations with clinical characteristics in a multicenter study. Respir Med 2012;106:522-30.

62. Garcia-Aymerich J, et al. Regular physical activity reduces hospital admission and mortality in chronic obstructive pulmonary disease: a population based cohort study. Thorax 2006;61:772-8.

63. Ringbaek TJ, Lange P. Outdoor activity and performance status as predictors of survival in hypoxaemic chronic obstructive pulmonary disease (COPD). Clin Rehabil 2005;19:331-8.

64. Waschki B, et al Physical activity is the strongest predictor of allcause mortality in patients with COPD: a prospective cohort study. Chest 2011;140:331-42.

65. Gimeno-Santos E. et al. The PROactive instruments to measure physical activity in patients with chronic obstructive pulmonary disease. Eur Respir J 2015;46:988-1000.

66. Gouzi F, et al. Evidence of an early physical activity reduction in chronic obstructive pulmonary disease patients. Arch Phys Med Rehabil 2011;92:1611-1617.

67. Garg PK, et al. Physical activity during daily life and mortality in patients with peripheral arterial disease. Circulation 2006;114:242-8.
68. Manini TM, et al. Daily activity energy expenditure and mortality among older adults. Jama 2006;296:171-9.

69. Garcia-Aymerich J, et al. Regular physical activity modifies smoking-related lung function decline and reduces risk of chronic obstructive pulmonary disease: a population-based cohort study. Am J Respir Crit Care Med 2007;175: 458-63.

70. Spruit MA, et al. Pulmonary rehabilitation and physical activity in patients with chronic obstructive pulmonary disease. Am J Respir Crit Care Med 2015;192:924-33.

71. Mantoani LC, et al. Interventions to modify physical activity in patients with COPD: a systematic review. Eur Respir J 2016;48:69-81.

72. Casaburi R. Activity promotion: a paradigm shift for chronic obstructive pulmonary disease therapeutics. Proc Am Thorac Soc 2011;8:334-7.

73. Cindy Ng LWC, et al. Does exercise training change physical activity in people with COPD? A systematic review and meta-analysis. Chron Respir Dis 2012;9:17-26

74. Zuwallack RL, Nici L. Modifying the course of chronic obstructive pulmonary disease: looking beyond the $\mathrm{FEV}_{1}$. COPD 2012;9:637-48.

75. Jencks SF, et al. Rehospitalizations among patients in the Medicare fee-for-service program. N Engl J Med 2009;360:1418-28.

76. Kaiser Health News. Younger seniors amass more end-of-life care than oldest americans, study finds. Available from: http//khn.org/ news/younger-seniors-amass-more-end-of-life-care-than-oldestamericans-study-finds/

77. Spencer S, Jones PW. Time course of recovery of health status following an infective exacerbation of chronic bronchitis. Thorax 2003;58:589-93.

78. Ehsan M, et al. A longitudinal study evaluating the effect of exacerbations on physical activity in patients with chronic obstructive pulmonary disease. Ann Am Thorac Soc 2013;10:559-64.

79. Suissa $\mathrm{S}$, et al. Long-term natural history of chronic obstructive pulmonary disease: severe exacerbations and mortality. Thorax 2012;67: 957-63.

80. Hurst JR, et al. Temporal clustering of exacerbations in chronic obstructive pulmonary disease. Am J Respir Crit Care Med 2009; 179: 369-74.

81. Chawla H, et al. Physical activity as a predictor of thirty-day hospital re-admission after a discharge for a clinical exacerbation of COPD. Ann Am Thorac Soc 2014;11:1203-9.

82. Bodenheimer T, et al. Improving primary care for patients with chronic illness. JAMA 2002;288:1775-9.

83. Nici L, et al. Self-management in chronic obstructive pulmonary disease. Time for a paradigm shift? Ann Am Thorac Soc 2014;11:101-7.

84. Boyd CM, et al, Clinical practice guidelines and quality of care for older patients with multiple comorbid diseases: implications for pay for performance. JAMA 2005;294:716-24.

85. Sin DD, et al. Mortality in COPD: role of comorbidities. Eur Respir J 2006;28:1245-57.

86. Bodenheimer T. Planned visits to help patients self-manage chronic conditions. Am Fam Physician 2005;72:1454-56.

87. Bodenheimer T, et al. Improving primary care for patients with chronic illness: the chronic care model, Part 2. JAMA, 2002;2881909-14.

88. Nici L, et al. An official American Thoracic Society workshop report: the Integrated Care of the COPD Patient. Proc Am Thorac Soc 2012; 9:9-18.

89. Valentijn PP, et al. Understanding integrated care: a comprehensive conceptual framework based on the integrative functions of primary care. Int J Integr Care 2013;13:e010.

90. Jennings $\mathrm{JH}$, et al. Predischarge bundle for patients with acute exacerbations of COPD to reduce readmissions and ED visits: a randomized controlled trial. Chest 2015;147:1227-34. 
91. Ospina MB, et al. A Delphi consensus study among clinicians to inform a discharge care bundle for chronic obstructive pulmonary disease. Am J Respir Crit Care Med 2016;193:A1107.

92. Jordan RE, et al. Supported self-management for patients with moderate to severe chronic obstructive pulmonary disease (COPD): an evidence synthesis and economic analysis. Health Technol Assess 2015;19:1-516.

93. Puhan MA, et al. Pulmonary rehabilitation following exacerbations of chronic obstructive pulmonary disease. Cochrane Database Syst Rev 2011: CD005305.

94. Benzo R, et al. Health coaching and chronic obstructive pulmonary disease rehospitalization: a randomized study. Am J Respir Crit Care Med 2016;194:672-80.

95. PCMH-AHRQ. Defining the PCMH. Available from: https://pcmh. ahrq.gov/page/defining-pcmh

96. Ortiz, G, Fromer L. Patient-centered medical home in chronic obstructive pulmonary disease. J Multidiscip Healthc 2011;4:357-65.

97. Wagner EH. Chronic disease management: what will it take to improve care for chronic illness? Eff Clin Pract 1998;1: 2-4.

98. Adams SG, et al. Systematic review of the chronic care model in chronic obstructive pulmonary disease prevention and management. Arch Intern Med 2007;167:551-61.

99. Wagg K, Unravelling self-management for COPD: what next? Chron Respir Dis 2012;9:5-7.

100. Boyd CM, et al. Guided care for multimorbid older adults. Gerontologist 2007;47:697-704.

101. Boyd CM, et al., The effects of guided care on the perceived quality of health care for multi-morbid older persons: 18-month outcomes from a cluster-randomized controlled trial. J Gen Intern Med 2010;25:235-42.

102. Leff B, et al. Guided care and the cost of complex healthcare: a preliminary report. Am J Manag Care 2009;15:555-9.

103. Boult C, et al. The effect of guided care teams on the use of health services: results from a cluster-randomized controlled trial. Arch Intern Med 2011;171:460-6.

104. Boult C, et al. A matched-pair cluster-randomized trial of guided care for high-risk older patients. J Gen Intern Med 2013;28:612-21.

105. Marsteller JA, et al, Effects of guided care on providers' satisfaction with care: a three-year matched-pair cluster-randomized trial. Popul Health Manag 2013;16:317-25.

106. Bourbeau J, et al. Reduction of hospital utilization in patients with chronic obstructive pulmonary disease: a disease-specific selfmanagement intervention. Arch Intern Med 2003;163:585-91.

107. Casas A, et al. Integrated care prevents hospitalisations for exacerbations in COPD patients. Eur Respir J 2006;28:123-30.

108. Rice KL, et al. Disease management program for chronic obstructive pulmonary disease: a randomized controlled trial. Am J Respir Crit Care Med 2010;182:890-6.

109. Centre NCG. Chronic obstructive pulmonary disease: Management of chronic obstructive pulmonary disease in adults in primary and secondary care, 2010. Available from: http://guidance. nice.org.uk/CG101/Guidance/pdf/English

110. Schunemann HJ, et al. A vision statement on guideline development for respiratory disease: the example of COPD. Lancet 2009;373:774-9.

111. New England Healthcare Institute. Improving physician adherence to clinical practice guidelines. Barriers and strategies for change. New England Healthcare Institute 2008;8:1-63.

112. British Thoracic Society. The role of the respiratory specialist in the provision of integrated care and long term conditions management, 2014. Available from: https:/www.brit-thoracic.org.uk/document-library/delivery-of-respiratory-care/integrated-care/bts-integrated-care-position-statement-october-2014
113. Kruis AL, et al. Cochrane corner: is integrated disease management for patients with COPD effective? Thorax 2014;69:1053-5.

114. Pinnock $\mathrm{H}$, et al. Effectiveness of telemonitoring integrated into existing clinical services on hospital admission for exacerbation of chronic obstructive pulmonary disease: researcher blind, multicentre, randomised controlled trial. BMJ 2013;347:f6070.

115. Addicott R. Commissioning and contracting for integrated care, 2014. Available from: http://www.kingsfund.org.uk/sites/files/kf/ kings-fund-commissioning-contracting-integrated-care-nov14.pdf

116. Bourbeau J, et al. Comprehensive Self-Management Strategies. Semin Respir Crit Care Med 2015;36:630-8.

117. Zwerink M, et al. Self management for patients with chronic obstructive pulmonary disease. Cochrane Database Syst Rev 2014:3:CD002990.

118. Bucknall CE, et al. Glasgow supported self-management trial (GSuST) for patients with moderate to severe COPD: randomised controlled trial. BMJ 2012;344:e1060.

119. Bischoff EW, et al. Effects of written action plan adherence on COPD exacerbation recovery. Thorax 2011;66:26-31.

120. Fan VS, et al.,A comprehensive care management program to prevent chronic obstructive pulmonary disease hospitalizations: a randomized, controlled trial. Ann Intern Med 2012;156: 673-83.

121. Criner GJ, et al. Prevention of acute exacerbations of COPD: American College of Chest Physicians and Canadian Thoracic Society Guideline. Chest 2015;147:894-942.

122. Bourbeau J, et al. An international randomized study of a homebased self-management program for severe COPD: the COMET. Int J Chron Obstruct Pulmon Dis 2016;11:1447-51.

123. Viegi G, et al. Definition, epidemiology and natural history of COPD. Eur Respir J 2007;30:993-1013.

124. Guerriero M, et al. COPD prevalence in a north-eastern Italian general population. Respir Med 2015;109:1040-7.

125. Cazzola M, et al, Prevalence of comorbidities in patients with chronic obstructive pulmonary disease. Respiration 2010;80: 112-9.

126. Pandolfi P, et al. Socio-economic and clinical factors as predictors of disease evolution and acute events in COPD patients. PLoS One 2015;10:e0135116.

127. Gini R, et al. Chronic disease prevalence from Italian administrative databases in the VALORE project: a validation through comparison of population estimates with general practice databases and national survey. BMC Public Health 2013;13:15.

128. Dal Negro RW, et al.,Costs of chronic obstructive pulmonary disease (COPD) in Italy: the SIRIO study (social impact of respiratory integrated outcomes). Respir Med 2008;102:92-101.

129. Dal Negro RW, et al. Costs of illness analysis in Italian patients with chronic obstructive pulmonary disease (COPD): an update. Clinicoecon Outcomes Res 2015;7:153-9.

130. Blasi F, et al. The clinical and economic impact of exacerbations of chronic obstructive pulmonary disease: a cohort of hospitalized patients. PLoS One 2014;9:e101228.

131. Barbato A, et al. [Tuscan chronic care model: a preliminary analysis].[Article in Italian]. Ig Sanita Pubbl 2015;71:499-513.

132. Sanguinetti CM, et al. Standards of suitability for the management of chronic obstructive respiratory diseases. Multidiscip Respir Med 2014; 9:65.

133. Nardini S, et al. Audit on the appropriateness of integrated COPD management: the "ALT-BPCO" project. Multidiscip Respir Med 2014;9:40.

134. Ministero della Salute. [D.G.P.S. Nazionale della Cronicità 2016] [in Italian]. Available from: http://www.salute.gov.it 
135. Maio S, et al. COPD management according to old and new GOLD guidelines: an observational study with Italian general practitioners. Curr Med Res Opin 2014;30:1033-42.

136. Bertella E, et al. COPD management in primary care: is an educational plan for GPs useful? Multidiscip Respir Med 2013;8:24.

137. ISTAT. Aspects of Daily life: health status. 2011; Available from: http://dati.istat.it/?lang $=$ en\&SubSessionId $=818 \mathrm{~d} 3 \mathrm{ada}-3 \mathrm{e} 9 \mathrm{~b}-$ 4a10-9e4f-d71358a5ba0a\&themetreeid=-200

138. Francesconi P, et al. [Primary care and chronic diseases: geographical differences in avoidable hospitalization]. [Article in Italian]. Epidemiol Prev 2011;35:128-9.

139. Spruit MA, et al. Differences in content and organizational aspects of pulmonary rehabilitation programs. Eur Respir J 2014;43: 1326-37.

140. Balbi B, et al. Pulmonary rehabilitation in Italy: professional barriers to overcome. Eur Respir J 2014;44:1382-3.

141. McLean S, et al. Telehealthcare for chronic obstructive pulmonary disease: Cochrane Review and meta-analysis. Br J Gen Pract 2012; 62:e739-49.

142. Paneroni M, et al. Feasibility and effectiveness of an educational program in Italian COPD patients undergoing rehabilitation. Respir Care 2013;58:327-33.

143. Bernocchi P, et al. Healthcare continuity from hospital to territory in Lombardy: TELEMACO project. Am J Manag Care 2012;18:e101-8.

144. Vitacca M, et al. Home-based telemanagement in advanced COPD: who uses it most? Real-life study in Lombardy. COPD 2016;13:491-8.

145. Vitacca M, et al. Is There any additional effect of tele-assistance on long-term care programmes in hypercapnic COPD patients? A retrospective study. COPD 2016;13:576-82.

146. Chatwin M, et al. Randomised crossover trial of telemonitoring in chronic respiratory patients (TeleCRAFT trial). Thorax 2016; 71: 305-11.

147. WHO. Global status report on non communicable diseases 2010. Available from: http://apps.who.int/iris/bitstream/10665/44579/1/ 9789240686458_eng.pdf

148. Han MK, et al. Chronic obstructive pulmonary disease phenotypes: the future of COPD. Am J Respir Crit Care Med 2010;182:598-604.

149. GOLD. [Global strategy for the diagnosis, management, and prevention of chronic obstructive pulmonary disease (revised 2015)].[In Italian]. Available from: http://goldcopd.it/wp-content/ uploads/materiali/2015/Gold_Pocket_DEF_2015.pdf

150. Hodgkin JE, et al. American Thoracic Society. Medical Section of the American Lung Association. Pulmonary rehabilitation. Am Rev Respir Dis 1981;124:663-6.

151. Lusuardi M, et al. Definition and rationale for pulmonary rehabilitation. In: C. Donner, N. Ambrosino and R. Goldstein (eds.), Pulmonary rehabilitation. 2005; Taylor \& Francis, Boca Raton; p. 3-8.

152. Fishman AP. Pulmonary rehabilitation research. Am J Respir Crit Care Med 1994:149: 825-33.

153. AARC. Clinical practice guideline on pulmonary rehabilitation, 2002 Available from: http://www.rcjournal.com/cpgs/prcpg.html

154. WHO. Preparing a healthcare workforce for the 21st century: the challenge of chronic conditions. Available from: http://www.who.int/ chp/knowledge/publications/workforce_report/en/
155. Spruit MA, et al. Integration of pulmonary rehabilitation in COPD. Lancet 2008;371:12-3.

156. 1Amelsfoort PV. [The design of work and organisation].[in Dutch]. 2000; ST-Groep Vlijmen.

157. Clegg CW, Sociotechnical principles for system design. Appl Ergon 2000;31:463-77.

158. Porter M, Lee T. The strategy that will fix health care. Harvard Business Review 2013. Available from: https://hbr.org/2013/10/ the-strategy-that-will-fix-health-care

159. Bohmer RM. The four habits of high-value health care organizations. N Engl J Med 2011;365:2045-7.

160. Huber M, et al, How should we define health? BMJ 2011;343: d4163.

161. Plsek PE, Wilson T. Complexity, leadership, and management in healthcare organisations. BMJ 2001;323:746-9.

162. Wilson T, et al. Complexity science: complexity and clinical care. BMJ 2001;323:685-8.

163. Bourbeau J. Preventing hospitalization for COPD exacerbations. Semin Respir Crit Care Med 2010;31:313-20.

\section{Appendix}

\section{Introduction (Claudio F. Donner)}

Prevalence of COPD (Laura Carrozzi, Sara Maio, Sandra Baldacci, Francesco Pistelli, Giovanni Viegi)

An overview of COPD (Andrea Purro)

COPD and chronic heart disease (Roberto Torchio)

Physical activity and long-term management of COPD patient (Enrico Clini)

The integrated care of the COPD patient (Sandro Amaducci, Richard L. ZuWallack)

Delivery system design and decision support (Roger Goldstein)

Integrated care for COPD: patients versus systems (Michael Morgan)

Self-management and health information technology

(Jean Bourbeau)

Integrated care for patients with COPD in Italy (Guido Vagheggini)

The CIRO+ Program: an integrated, holistic, personalized medical strategy (Emiel F.M. Wouters)

Medical management of the at-risk COPD patient

(Richard L. ZuWallack) 